This item was submitted to Loughborough's Research Repository by the author.

Items in Figshare are protected by copyright, with all rights reserved, unless otherwise indicated.

\title{
G- and Go-hypoellipticity of partial differential operators with constant Colombeau coefficients
}

PLEASE CITE THE PUBLISHED VERSION

http://dx.doi.org/10.4064/bc88

PUBLISHER

(C) IMPAN

VERSION

AM (Accepted Manuscript)

\section{PUBLISHER STATEMENT}

This work is made available according to the conditions of the Creative Commons Attribution-NonCommercialNoDerivatives 4.0 International (CC BY-NC-ND 4.0) licence. Full details of this licence are available at: https://creativecommons.org/licenses/by-nc-nd/4.0/

\section{LICENCE}

CC BY-NC-ND 4.0

\section{REPOSITORY RECORD}

Garetto, Claudia. 2019. "G- and Gळ-hypoellipticity of Partial Differential Operators with Constant Colombeau Coefficients". figshare. https://hdl.handle.net/2134/17316. 


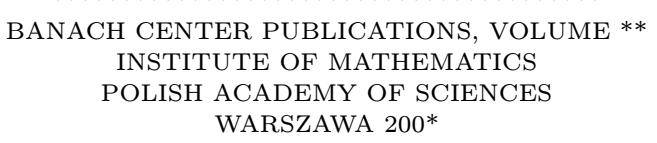

\title{
$\mathcal{G}$ AND $\mathcal{G}^{\infty}$-HYPOELLIPTICITY \\ OF PARTIAL DIFFERENTIAL OPERATORS WITH CONSTANT COLOMBEAU COEFFICIENTS
}

\author{
CLAUDIA GARETTO \\ Institut für Grundlagen der Bauingenieurwissenschaften \\ Leopold-Franzens-Universität Innsbruck \\ Technikerstr. 13, A 6020 Innsbruck, Austria \\ E-mail: claudia@mat1.uibk.ac.at
}

\begin{abstract}
We provide a deep investigation of the notions of $\mathcal{G}$ - and $\mathcal{G}^{\infty}$-hypoellipticity for partial differential operators with constant Colombeau coefficients. This involves generalized polynomials and fundamental solutions in the dual of a Colombeau algebra. Sufficient conditions and necessary conditions for $\mathcal{G}$ - and $\mathcal{G}^{\infty}$-hypoellipticity are given.
\end{abstract}

1. Introduction. Over the past thirty years, nonlinear theories of generalized functions have been developed by many authors $[1,15,19,20]$ mainly inspired by the work of $\mathrm{J}$. F. Colombeau [2]. They have proved to be a valuable tool for treating partial differential equations with singular data or coefficients $[3,12,17,20]$. Recently, intense research has been done in the context of partial differential operators with generalized coefficients, leading to the development of a theory of generalized Fourier and pseudodifferential operators acting on Colombeau algebras $[6,12,14,17]$ as well as to microlocal analysis in the Colombeau context $[9,13,18]$. Increasing importance is also attached to an understanding of topological structures $[4,5,7,8]$ in spaces of generalized functions and to the development of functional analytic methods [10].

Starting from an investigation of the mapping properties of partial differential operators with Colombeau coefficients, many authors have given particular attention to the notion of generalized hypoellipticity. This is related to a regularity theory for Colombeau generalized functions, based in the usual Colombeau algebra $\mathcal{G}(\Omega)$ on the subalgebra $\mathcal{G}^{\infty}(\Omega)$ and consistent with the classical $\mathcal{C}^{\infty}$-regularity in the distributional context. Indeed, $\mathcal{G}^{\infty}(\Omega) \cap \mathcal{D}^{\prime}(\Omega)=\mathcal{C}^{\infty}(\Omega)[20]$. By means of pseudodifferential techniques elaborated

2000 Mathematics Subject Classification: Primary 46F30; Secondary 35 H99.

Key words and phrases: Colombeau algebras, hypoellipticity.

Research of the author supported by FWF (Austria), grant T305-N13.

The paper is in final form and no version of it will be published elsewhere. 
in $[6,12,13]$, sufficient conditions of $\mathcal{G}^{\infty}$-hypoellipticity has been provided involving the symbol of the operator $P(x, D)=\sum_{|\alpha| \leq m} c_{\alpha}(x) D^{\alpha}, c_{\alpha} \in \mathcal{G}(\Omega)$, or more in general the symbol of a generalized pseudodifferential operator [12, 13, 17, 18]. These are conditions which allow to deduce from $P(x, D) u \in \mathcal{G}^{\infty}(\Omega)$ that the generalized function $u$ belongs to $\mathcal{G}^{\infty}(\Omega)$ as well.

The development of a topological theory of spaces of Colombeau type $[5,7,8]$ is deeply connected to the more general treatment of locally convex topological $\widetilde{\mathbb{C}}$-modules, where $\widetilde{\mathbb{C}}$ is the ring of complex generalized numbers, and to a duality theory in this setting. The duals of the Colombeau algebras $\mathcal{G}_{\mathrm{c}}(\Omega)$ and $\mathcal{G}(\Omega)$, i.e., the spaces $\mathcal{L}\left(\mathcal{G}_{\mathrm{c}}(\Omega), \widetilde{\mathbb{C}}\right)$ and $\mathcal{L}(\mathcal{G}(\Omega), \widetilde{\mathbb{C}})$ of all $\widetilde{\mathbb{C}}$-linear and continuous functionals on $\mathcal{G}_{\mathrm{c}}(\Omega)$ and $\mathcal{G}(\Omega)$ respectively, play a main role in the kernel theory for generalized Fourier and pseudodifferential operators $[4,12,13,14]$. In particular, one can now shift issues of solvability, regularity theory and microlocal analysis from the level of generalized functions to the level of $\widetilde{\mathbb{C}}$-linear functionals.

Since $\mathcal{L}\left(\mathcal{G}_{\mathrm{c}}(\Omega), \widetilde{\mathbb{C}}\right)$ contains both $\mathcal{G}^{\infty}(\Omega)$ and $\mathcal{G}(\Omega)$, two levels of regularity concern a functional in $\mathcal{L}\left(\mathcal{G}_{\mathrm{c}}(\Omega), \widetilde{\mathbb{C}}\right)$ : the regularity with respect to $\mathcal{G}(\Omega)$ and the regularity with respect to $\mathcal{G}^{\infty}(\Omega)$. This leads to two different notions of generalized hypoellipticity in the dual $\mathcal{L}\left(\mathcal{G}_{\mathrm{c}}(\Omega), \widetilde{\mathbb{C}}\right)$ : the $\mathcal{G}^{\infty}$-hypoellipticity, consistent with the already existent notion on $\mathcal{G}(\Omega)$, and the new $\mathcal{G}$-hypoellipticity. The investigation of $\mathcal{G}^{\infty}$ - and $\mathcal{G}$-hypoellipticity is mainly concentrated on those functionals $T$ which have basic structure, or in other words admit a representing net $\left(T_{\varepsilon}\right)_{\varepsilon}$. In [9] the author has achieved conditions on the symbol of a generalized pseudodifferential operator $P(x, D)$ which are sufficient for its $\mathcal{G}^{\infty}$ - or $\mathcal{G}_{\text {- }}$ hypoellipticity. This means that if $P(x, D) T \in \mathcal{G}(\Omega)\left(\operatorname{resp} . \mathcal{G}^{\infty}(\Omega)\right)$ and $T \in \mathcal{L}\left(\mathcal{G}_{\mathrm{c}}(\Omega), \widetilde{\mathbb{C}}\right)$ is basic then it is actually an element of $\mathcal{G}(\Omega)\left(\operatorname{resp} . \mathcal{G}^{\infty}(\Omega)\right)$.

The search for necessary conditions for $\mathcal{G}$ - and $\mathcal{G}^{\infty}$-hypoellipticity has been a longstanding open problem. The first necessary condition for $\mathcal{G}^{\infty}$-hypoellipticity of partial differential operators with constant Colombeau coefficients has be obtained in [10] by making use of a generalized version of the closed graph theorem valid for Fréchet $\widetilde{\mathbb{C}}$-modules. This is one of the first applications of functional analytic methods in the Colombeau context. The $\mathcal{G}$-part of the necessary conditions' problem has been open until [11]. Crucial has been the introduction of a notion of fundamental solution as a functional in the dual $\mathcal{L}\left(\mathcal{G}_{\mathrm{c}}\left(\mathbb{R}^{n}\right), \widetilde{\mathbb{C}}\right)$. This has lead in [11] to characterizations of $\mathcal{G}$ - and $\mathcal{G}^{\infty}$-hypoellipticity which relay on the $\mathcal{G}$ - or $\mathcal{G}^{\infty}$-behaviour of a fundamental solution outside the origin.

The aim of this paper is to investigate the notions of $\mathcal{G}$-and $\mathcal{G}^{\infty}$-hypoellipticity for partial differential operators with constant Colombeau coefficients, adding some new results to what is already known on this topic. By making use of concepts as generalized polynomials and fundamental solutions in $\mathcal{L}\left(\mathcal{G}_{\mathrm{c}}\left(\mathbb{R}^{n}\right), \widetilde{\mathbb{C}}\right)$, we avoid those pseudodifferential techniques which become essential when the coefficients are not constant. In this way, our treatment of $\mathcal{G}$ - and $\mathcal{G}^{\infty}$-hypoellipticity for partial differential operators with constant Colombeau coefficients stays closer to the well-known Hörmander investigation for classical operators.

We now describe the contents of the sections.

Section 2 collects the necessary background of Colombeau theory. Section 3, modelled 
on $[21$, Section 2.1], approaches the general problem of hypoellipticity in the wider context of continuous $\widetilde{\mathbb{C}}$-linear maps $k$ acting from $\mathcal{G}_{\mathrm{c}}\left(\Omega^{\prime}\right)$ to $\mathcal{L}\left(\mathcal{G}_{\mathrm{c}}(\Omega), \widetilde{\mathbb{C}}\right)$, where $\Omega^{\prime}$ and $\Omega$ are open subsets of $\mathbb{R}^{n^{\prime}}$ and $\mathbb{R}^{n}$ respectively. As a particular case one can take $\Omega^{\prime}=\Omega=\mathbb{R}^{n}$ and $k=P(D)$. The remaining Sections 4,5 and 6 are devoted to partial differential operators with constant Colombeau coefficients. We begin with some characterizations and necessary conditions for $\mathcal{G}$ - and $\mathcal{G}^{\infty}$-hypoellipticity in Section 4 . In addition, we improve the results of Theorem 4.9 of [11] in Theorem 15 and we obtain some new sufficient conditions in Section 5, Theorem 17 and Proposition 18. These conditions are employed in Section 6 in proving that $\mathcal{G}$ - and $\mathcal{G}^{\infty}$-elliptic operators are $\mathcal{G}$ - and $\mathcal{G}^{\infty}$ hypoelliptic, respectively.

\section{Basic notions.}

2.1 Colombeau theory: topology and duality theory.

In the sequel we make use of the following concept of slow scale net (s.s.n). A slow scale net is a net $\left(r_{\varepsilon}\right)_{\varepsilon} \in \mathbb{C}^{(0,1]}$ such that

$$
\forall q \geq 0 \exists c_{q}>0 \forall \varepsilon \in(0,1] \quad\left|r_{\varepsilon}\right|^{q} \leq c_{q} \varepsilon^{-1} .
$$

Colombeau model. As pointed out in $[7,8]$ the most common spaces and algebras of generalized functions of Colombeau type can be introduced and investigated under a topological point of view by making use of the following models.

Let $E$ be a locally convex topological vector space topologized through the family of seminorms $\left\{p_{i}\right\}_{i \in I}$. The elements of

$$
\begin{gathered}
\mathcal{M}_{E}:=\left\{\left(u_{\varepsilon}\right)_{\varepsilon} \in E^{(0,1]}: \forall i \in I \exists N \in \mathbb{N} \quad p_{i}\left(u_{\varepsilon}\right)=O\left(\varepsilon^{-N}\right) \text { as } \varepsilon \rightarrow 0\right\}, \\
\mathcal{M}_{E}^{\text {sc }}:=\left\{\left(u_{\varepsilon}\right)_{\varepsilon} \in E^{(0,1]}: \forall i \in I \exists\left(\omega_{\varepsilon}\right)_{\varepsilon} \text { s.s.n. } \quad p_{i}\left(u_{\varepsilon}\right)=O\left(\omega_{\varepsilon}\right) \text { as } \varepsilon \rightarrow 0\right\}, \\
\mathcal{M}_{E}^{\infty}:=\left\{\left(u_{\varepsilon}\right)_{\varepsilon} \in E^{(0,1]}: \exists N \in \mathbb{N} \forall i \in I \quad p_{i}\left(u_{\varepsilon}\right)=O\left(\varepsilon^{-N}\right) \text { as } \varepsilon \rightarrow 0\right\}, \\
\mathcal{N}_{E}:=\left\{\left(u_{\varepsilon}\right)_{\varepsilon} \in E^{(0,1]}: \forall i \in I \forall q \in \mathbb{N} \quad p_{i}\left(u_{\varepsilon}\right)=O\left(\varepsilon^{q}\right) \text { as } \varepsilon \rightarrow 0\right\},
\end{gathered}
$$

are called $E$-moderate, $E$-moderate of slow scale type, $E$-regular and $E$-negligible, respectively. We define the space of generalized functions based on $E$ as the factor space $\mathcal{G}_{E}:=\mathcal{M}_{E} / \mathcal{N}_{E}$.

Real and complex generalized numbers. The rings $\widetilde{\mathbb{C}}=\mathcal{E}_{M} / \mathcal{N}$ of complex generalized numbers and $\widetilde{\mathbb{R}}$ of real generalized numbers are obtained by taking $E=\mathbb{C}$ and $E=\mathbb{R}$ respectively. $\widetilde{\mathbb{R}}$ can be endowed with some more structure by defining the order relation: $r \leq s$ if and only if there are representatives $\left(r_{\varepsilon}\right)_{\varepsilon},\left(s_{\varepsilon}\right)_{\varepsilon}$ with $r_{\varepsilon} \leq s_{\varepsilon}$ for all $\varepsilon$. It follows that $r \in \widetilde{\mathbb{R}}$ is positive $(r \geq 0)$ if there exists a representative $\left(r_{\varepsilon}\right)_{\varepsilon}$ such that $r_{\varepsilon} \geq 0$ for all $\varepsilon \in(0,1]$. An element $r$ of $\widetilde{\mathbb{R}}$ is called strictly nonzero if there exists some representative $\left(r_{\varepsilon}\right)_{\varepsilon}$ and an $m \in \mathbb{N}$ such that $\left|r_{\varepsilon}\right| \geq \varepsilon^{m}$ for all sufficiently small $\varepsilon$. Finally a positive and strictly nonzero $r \in \widetilde{\mathbb{R}}$ is called strictly positive. This means that $r_{\varepsilon} \geq \varepsilon^{m}$ for some representative $\left(r_{\varepsilon}\right)_{\varepsilon}$, some $m \in \mathbb{N}$ and for all $\varepsilon$ small enough.

Topological structure. For any locally convex topological vector space $E$ the space $\mathcal{G}_{E}$ has the structure of a $\widetilde{\mathbb{C}}$-module. The $\mathbb{C}$-module $\mathcal{G}_{E}^{\text {sc }}:=\mathcal{M}_{E}^{\text {sc }} / \mathcal{N}_{E}$ of generalized functions of slow scale type and the $\widetilde{\mathbb{C}}$-module $\mathcal{G}_{E}^{\infty}:=\mathcal{M}_{E}^{\infty} / \mathcal{N}_{E}$ of regular generalized functions are 
subrings of $\mathcal{G}_{E}$ with more refined assumptions of moderateness at the level of representatives. We use the notation $u=\left[\left(u_{\varepsilon}\right)_{\varepsilon}\right]$ for the class $u$ of $\left(u_{\varepsilon}\right)_{\varepsilon}$ in $\mathcal{G}_{E}$. This is the usual way adopted in the paper to denote an equivalence class.

The family of seminorms $\left\{p_{i}\right\}_{i \in I}$ on $E$ determines a locally convex $\widetilde{\mathbb{C}}$-linear topology on $\mathcal{G}_{E}$ (see [7, Definition 1.6]) by means of the valuations

$$
\mathrm{v}_{p_{i}}\left(\left[\left(u_{\varepsilon}\right)_{\varepsilon}\right]\right):=\mathrm{v}_{p_{i}}\left(\left(u_{\varepsilon}\right)_{\varepsilon}\right):=\sup \left\{b \in \mathbb{R}: \quad p_{i}\left(u_{\varepsilon}\right)=O\left(\varepsilon^{b}\right)\right\}
$$

and the corresponding ultra-pseudo-seminorms $\left\{\mathcal{P}_{i}\right\}_{i \in I}$. The theoretical presentation concerning definitions and properties of valuations and ultra-pseudo-seminorms in the abstract context of $\widetilde{\mathbb{C}}$-modules is here omitted for the sake of brevity and can be found in [7, Subsections 1.1, 1.2].

In the current paper the valuation and the ultra-pseudo-norm on $\widetilde{\mathbb{C}}$ obtained through the absolute value in $\mathbb{C}$ are denoted by $\mathrm{v}$ and $|\cdot|_{\mathrm{e}}$ respectively. The Colombeau algebra $\mathcal{G}(\Omega)=\mathcal{E}_{M}(\Omega) / \mathcal{N}(\Omega)$ is obtained as a $\widetilde{\mathbb{C}}$-module of $\mathcal{G}_{E}$-type by choosing $E=\mathcal{E}(\Omega)$. The seminorms $p_{K, i}(f)=\sup _{x \in K,|\alpha| \leq i}\left|\partial^{\alpha} f(x)\right|$, where $K$ is a compact subset of $\Omega$, generate the family of ultra-pseudo-seminorms $\mathcal{P}_{K, i}(u)=\mathrm{e}^{-\mathrm{v}_{p_{K, i}}(u)}$ and give to $\mathcal{G}(\Omega)$ the topological structure of a Fréchet $\widetilde{\mathbb{C}}$-module, i.e., of a complete and metrizable locally convex topological $\widetilde{\mathbb{C}}$-module. We recall that $\Omega \rightarrow \mathcal{G}(\Omega)$ is a fine sheaf of differential algebras on $\mathbb{R}^{n}$ and that the constants of $\mathcal{G}\left(\mathbb{R}^{n}\right)$ are the elements of $\widetilde{\mathbb{C}}$.

The Colombeau algebra $\mathcal{G}_{\mathrm{c}}(\Omega)$ of generalized functions with compact support is topologized by means of a strict inductive limit procedure. More precisely, setting $\mathcal{G}_{K}(\Omega):=$ $\left\{u \in \mathcal{G}_{\mathrm{c}}(\Omega): \operatorname{supp} u \subseteq K\right\}$ for $K \Subset \Omega, \mathcal{G}_{\mathrm{c}}(\Omega)$ is the strict inductive limit of the sequence of locally convex topological $\widetilde{\mathbb{C}}$-modules $\left(\mathcal{G}_{K_{n}}(\Omega)\right)_{n \in \mathbb{N}}$, where $\left(K_{n}\right)_{n \in \mathbb{N}}$ is an exhausting sequence of compact subsets of $\Omega$ such that $K_{n} \subseteq K_{n+1}$. We recall that the space $\mathcal{G}_{K}(\Omega)$ is endowed with the topology induced by $\mathcal{G}_{\mathcal{D}_{K^{\prime}}(\Omega)}$ where $K^{\prime}$ is a compact subset containing $K$ in its interior. In detail we consider on $\mathcal{G}_{K}(\Omega)$ the ultra-pseudo-seminorms $\mathcal{P}_{\mathcal{G}_{K}(\Omega), n}(u)=\mathrm{e}^{-\mathrm{v}_{K, n}(u)}$. Note that the valuation $\mathrm{v}_{K, n}(u):=\mathrm{v}_{p_{K^{\prime}, n}}(u)$ is independent of the choice of $K^{\prime}$ when acts on $\mathcal{G}_{K}(\Omega)$.

Regularity theory. Regularity theory in the Colombeau context as initiated in [20] is based on the subalgebra $\mathcal{G}^{\infty}(\Omega)=\mathcal{E}_{M}^{\infty}(\Omega) / \mathcal{N}(\Omega)$ of $\mathcal{G}(\Omega)$ obtained as $\mathcal{G}_{E}^{\infty}$-space when $E=\mathcal{E}(\Omega)$. The intersection of $\mathcal{G}^{\infty}(\Omega)$ with $\mathcal{G}_{\mathrm{c}}(\Omega)$ defines $\mathcal{G}_{\mathrm{c}}^{\infty}(\Omega)$. We finally consider the Colombeau algebras $\mathcal{G}_{\mathscr{S}}\left(\mathbb{R}^{n}\right)=\mathcal{E}_{\mathscr{S}}\left(\mathbb{R}^{n}\right) / \mathcal{N}_{\mathscr{S}}\left(\mathbb{R}^{n}\right)$ and $\mathcal{G}_{\mathscr{S}}^{\infty}\left(\mathbb{R}^{n}\right)=\mathcal{E}_{\mathscr{S}}^{\infty}\left(\mathbb{R}^{n}\right) / \mathcal{N}_{\mathscr{S}}\left(\mathbb{R}^{n}\right)$ of generalized functions based on $\mathscr{S}\left(\mathbb{R}^{n}\right)$ determined as $\mathcal{G}_{E}$ and $\mathcal{G}_{E}^{\infty}$ spaces respectively by taking $E=\mathscr{S}\left(\mathbb{R}^{n}\right)$. From a topological point of view $\mathcal{G}_{\mathscr{S}}\left(\mathbb{R}^{n}\right)$ and $\mathcal{G}^{\infty}(\Omega)$ are Fréchet $\widetilde{\mathbb{C}}$-modules, $\mathcal{G}_{\mathrm{c}}^{\infty}(\Omega)$ is the strict inductive limit of a family of ultra-pseudo-normed $\widetilde{\mathbb{C}}$ modules and $\mathcal{G}_{\mathscr{S}}^{\infty}\left(\mathbb{R}^{n}\right)$ is an ultra-pseudo-normed $\widetilde{\mathbb{C}}$-module.

Duality theory. A duality theory for $\widetilde{\mathbb{C}}$-modules had been developed in $[7,8]$ in the framework of topological and locally convex topological $\widetilde{\mathbb{C}}$-modules. Starting from an investigation of $\mathcal{L}(\mathcal{G}, \widetilde{\mathbb{C}})$, the $\widetilde{\mathbb{C}}$-module of all $\widetilde{\mathbb{C}}$-linear and continuous functionals on $\mathcal{G}$, it provides the theoretical tools for dealing with the topological duals of the Colombeau algebras $\mathcal{G}_{\mathrm{c}}(\Omega), \mathcal{G}(\Omega)$ and $\mathcal{G}_{\mathscr{S}}\left(\mathbb{R}^{n}\right)$. The spaces $\mathcal{L}(\mathcal{G}(\Omega), \widetilde{\mathbb{C}}), \mathcal{L}\left(\mathcal{G}_{\mathrm{c}}(\Omega), \widetilde{\mathbb{C}}\right)$ and $\mathcal{L}\left(\mathcal{G}_{\mathscr{S}}\left(\mathbb{R}^{n}\right), \widetilde{\mathbb{C}}\right)$ are endowed with the topology of uniform convergence on bounded subsets (see [7, Remark 2.11]) and, as proven in $[8$, Theorems 3.1, 3.8], the following chains of continuous embeddings 
hold:

$$
\begin{gathered}
\mathcal{G}^{\infty}(\Omega) \subseteq \mathcal{G}(\Omega) \subseteq \mathcal{L}\left(\mathcal{G}_{\mathrm{c}}(\Omega), \widetilde{\mathbb{C}}\right), \\
\mathcal{G}_{\mathrm{c}}^{\infty}(\Omega) \subseteq \mathcal{G}_{\mathrm{c}}(\Omega) \subseteq \mathcal{L}(\mathcal{G}(\Omega), \widetilde{\mathbb{C}}), \\
\mathcal{G}_{\mathscr{S}}^{\infty}\left(\mathbb{R}^{n}\right) \subseteq \mathcal{G}_{\mathscr{S}}\left(\mathbb{R}^{n}\right) \subseteq \mathcal{L}\left(\mathcal{G}_{\mathscr{S}}\left(\mathbb{R}^{n}\right), \widetilde{\mathbb{C}}\right) .
\end{gathered}
$$

Since $\Omega \rightarrow \mathcal{L}\left(\mathcal{G}_{\mathrm{c}}(\Omega), \widetilde{\mathbb{C}}\right)$ is a sheaf we can define the support of a functional $T$ (denoted by supp $T)$. In analogy with distribution theory from Theorem 1.2 in $[8]$ we have that $\mathcal{L}(\mathcal{G}(\Omega), \widetilde{\mathbb{C}})$ can be identified with the set of functionals in $\mathcal{L}\left(\mathcal{G}_{\mathrm{c}}(\Omega), \widetilde{\mathbb{C}}\right)$ having compact support.

Regularity theory in the dual and basic structure. As already observed in $[9,14]$ the chains of inclusions in (1) make it meaningful to measure the regularity of a functional in $\mathcal{L}\left(\mathcal{G}_{\mathrm{c}}(\Omega), \widetilde{\mathbb{C}}\right)$ with respect to the algebras $\mathcal{G}(\Omega)$ and $\mathcal{G}^{\infty}(\Omega)$. We define the $\mathcal{G}$-singular

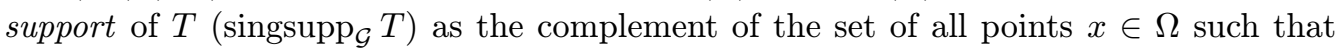
the restriction of $T$ to some open neighborhood $V$ of $x$ belongs to $\mathcal{G}(V)$. Analogously replacing $\mathcal{G}$ with $\mathcal{G}^{\infty}$ we introduce the notion of $\mathcal{G}^{\infty}$-singular support of $T$ denoted by $\operatorname{singsupp}_{\mathcal{G} \infty} T$. A microlocal analysis in the double $\mathcal{G}$ - and $\mathcal{G}^{\infty}$-version has been developed in the dual $\mathcal{L}\left(\mathcal{G}_{\mathrm{c}}(\Omega), \widetilde{\mathbb{C}}\right)$ by making use of the notions of $\mathcal{G}$ - and $\mathcal{G}^{\infty}$-wave front set [9]. In this context a main role is played by the functionals in $\mathcal{L}\left(\mathcal{G}_{\mathrm{c}}(\Omega), \widetilde{\mathbb{C}}\right)$ and $\mathcal{L}(\mathcal{G}(\Omega), \widetilde{\mathbb{C}})$ which have a "basic" structure. In detail, we say that $T \in \mathcal{L}\left(\mathcal{G}_{\mathrm{c}}(\Omega), \widetilde{\mathbb{C}}\right)$ is basic if there exists a net $\left(T_{\varepsilon}\right)_{\varepsilon} \in \mathcal{D}^{\prime}(\Omega)^{(0,1]}$ fulfilling the following condition: for all $K \Subset \Omega$ there exist $j \in \mathbb{N}, c>0, N \in \mathbb{N}$ and $\eta \in(0,1]$ such that

$$
\forall f \in \mathcal{C}_{K}^{\infty}(\Omega) \forall \varepsilon \in(0, \eta] \quad\left|T_{\varepsilon}(f)\right| \leq c \varepsilon^{-N} \sup _{x \in K,|\alpha| \leq j}\left|\partial^{\alpha} f(x)\right|
$$

and $T u=\left[\left(T_{\varepsilon} u_{\varepsilon}\right)_{\varepsilon}\right]$ for all $u \in \mathcal{G}_{\mathrm{c}}(\Omega)$.

In the same way a functional $T \in \mathcal{L}(\mathcal{G}(\Omega), \widetilde{\mathbb{C}})$ is said to be basic if there exists a net $\left(T_{\varepsilon}\right)_{\varepsilon} \in \mathcal{E}^{\prime}(\Omega)^{(0,1]}$ such that there exist $K \Subset \Omega, j \in \mathbb{N}, c>0, N \in \mathbb{N}$ and $\eta \in(0,1]$ with the property

$$
\forall f \in \mathcal{C}^{\infty}(\Omega) \forall \varepsilon \in(0, \eta] \quad\left|T_{\varepsilon}(f)\right| \leq c \varepsilon^{-N} \sup _{x \in K,|\alpha| \leq j}\left|\partial^{\alpha} f(x)\right|
$$

and $T u=\left[\left(T_{\varepsilon} u_{\varepsilon}\right)_{\varepsilon}\right]$ for all $u \in \mathcal{G}(\Omega)$.

Clearly the sets $\mathcal{L}_{\mathrm{b}}\left(\mathcal{G}_{\mathrm{c}}(\Omega), \widetilde{\mathbb{C}}\right)$ and $\mathcal{L}_{\mathrm{b}}(\mathcal{G}(\Omega), \widetilde{\mathbb{C}})$ of basic functionals are $\widetilde{\mathbb{C}}$-linear subspaces of $\mathcal{L}\left(\mathcal{G}_{\mathrm{c}}(\Omega), \widetilde{\mathbb{C}}\right)$ and $\mathcal{L}(\mathcal{G}(\Omega), \widetilde{\mathbb{C}})$ respectively.

2.2 Convolution between generalized functions and functionals, Fourier and Fourier-Laplace transform. We recall definition and properties of the convolution product between functionals and Colombeau generalized functions which are employed in the course of the paper. Detailed proofs can be found in [9, Section 1].

Proposition 1. The $\widetilde{\mathbb{C}}$-bilinear map

$$
(S, T) \rightarrow S * T:=S_{x} T_{y}(\cdot(x+y))
$$

(i) from $\mathcal{G}_{\mathrm{c}}\left(\mathbb{R}^{n}\right) \times \mathcal{L}_{\mathrm{b}}\left(\mathcal{G}_{\mathrm{c}}\left(\mathbb{R}^{n}\right), \widetilde{\mathbb{C}}\right)$ into $\mathcal{G}\left(\mathbb{R}^{n}\right)$,

(ii) from $\mathcal{G}\left(\mathbb{R}^{n}\right) \times \mathcal{L}_{\mathrm{b}}\left(\mathcal{G}\left(\mathbb{R}^{n}\right)\right.$, $\left.\widetilde{\mathbb{C}}\right)$ into $\mathcal{G}\left(\mathbb{R}^{n}\right)$, 
(iii) from $\mathcal{G}_{\mathscr{S}}\left(\mathbb{R}^{n}\right) \times \mathcal{L}_{\mathrm{b}}\left(\mathcal{G}\left(\mathbb{R}^{n}\right), \widetilde{\mathbb{C}}\right)$ into $\mathcal{G}_{\mathscr{S}}\left(\mathbb{R}^{n}\right)$,

(iv) from $\mathcal{G}_{\mathrm{c}}^{\infty}\left(\mathbb{R}^{n}\right) \times \mathcal{L}_{\mathrm{b}}\left(\mathcal{G}_{\mathrm{c}}\left(\mathbb{R}^{n}\right), \widetilde{\mathbb{C}}\right)$ into $\mathcal{G}^{\infty}\left(\mathbb{R}^{n}\right)$,

(v) from $\mathcal{G}^{\infty}\left(\mathbb{R}^{n}\right) \times \mathcal{L}_{\mathrm{b}}\left(\mathcal{G}\left(\mathbb{R}^{n}\right), \widetilde{\mathbb{C}}\right)$ into $\mathcal{G}^{\infty}\left(\mathbb{R}^{n}\right)$,

(vi) from $\mathcal{G}_{\mathscr{S}}^{\infty}\left(\mathbb{R}^{n}\right) \times \mathcal{L}_{\mathrm{b}}\left(\mathcal{G}\left(\mathbb{R}^{n}\right), \widetilde{\mathbb{C}}\right)$ into $\mathcal{G}_{\mathscr{S}}^{\infty}\left(\mathbb{R}^{n}\right)$,

(vii) from $\mathcal{L}\left(\mathcal{G}\left(\mathbb{R}^{n}\right), \widetilde{\mathbb{C}}\right) \times \mathcal{L}_{\mathrm{b}}\left(\mathcal{G}_{\mathrm{c}}\left(\mathbb{R}^{n}\right), \widetilde{\mathbb{C}}\right)$ into $\mathcal{L}\left(\mathcal{G}_{\mathrm{c}}\left(\mathbb{R}^{n}\right), \widetilde{\mathbb{C}}\right)$,

(viii) from $\mathcal{L}\left(\mathcal{G}_{\mathrm{c}}\left(\mathbb{R}^{n}\right), \widetilde{\mathbb{C}}\right) \times \mathcal{L}_{\mathrm{b}}\left(\mathcal{G}\left(\mathbb{R}^{n}\right), \widetilde{\mathbb{C}}\right)$ into $\mathcal{L}\left(\mathcal{G}_{\mathrm{c}}\left(\mathbb{R}^{n}\right), \widetilde{\mathbb{C}}\right)$,

(ix) from $\mathcal{L}\left(\mathcal{G}\left(\mathbb{R}^{n}\right), \widetilde{\mathbb{C}}\right) \times \mathcal{L}_{\mathrm{b}}\left(\mathcal{G}\left(\mathbb{R}^{n}\right), \widetilde{\mathbb{C}}\right)$ into $\mathcal{L}\left(\mathcal{G}\left(\mathbb{R}^{n}\right), \widetilde{\mathbb{C}}\right)$

is separately continuous.

Clearly, when $S$ and $T$ have both basic structure then the functional $S * T$ has basic structure too. Let now $\iota_{d}$ denote the embedding of $\mathcal{D}^{\prime}(\Omega)$ into the dual $\mathcal{L}\left(\mathcal{G}_{\mathrm{c}}(\Omega), \widetilde{\mathbb{C}}\right)$ given by $\iota_{d}(w)(u)=\left[\left(w\left(u_{\varepsilon}\right)\right)_{\varepsilon}\right]$, where $\left(u_{\varepsilon}\right)_{\varepsilon}$ is a representative of $u$ having support contained in a compact set uniformly with respect to $\varepsilon$. For any functional $T \in \mathcal{L}\left(\mathcal{G}_{\mathrm{c}}\left(\mathbb{R}^{n}\right), \widetilde{\mathbb{C}}\right)$ we have

$$
T * \iota_{d}(\delta)=T \text {. }
$$

The Colombeau algebra $\mathcal{G}_{\mathscr{S}}\left(\mathbb{R}^{n}\right)$ and its dual $\mathcal{L}\left(\mathcal{G}_{\mathscr{S}}\left(\mathbb{R}^{n}\right), \widetilde{\mathbb{C}}\right)$ are the natural setting where to define the Fourier transform $\mathcal{F}$ and its inverse $\mathcal{F}^{-1}$. In detail we employ the classical definition at the level of representatives in $\mathcal{G}_{\mathscr{S}}\left(\mathbb{R}^{n}\right)$ and the definition $\mathcal{F}(T)(u)=T(\mathcal{F}(u))$ on the functionals $T$ of $\mathcal{L}\left(\mathcal{G}_{\mathscr{S}}\left(\mathbb{R}^{n}\right), \widetilde{\mathbb{C}}\right)$. The reader may refer to [9, Subsection 1.4] for further explanation. In this paper, we will make use of the Fourier-Laplace transform of a generalized function in $\mathcal{G}_{\mathrm{c}}\left(\mathbb{R}^{n}\right)$. The following proposition shows that the classical Fourier-Laplace transform at the level of representatives allows to define the FourierLaplace transform of a generalized function in $\mathcal{G}_{\mathrm{c}}\left(\mathbb{R}^{n}\right)$ as a Colombeau object, more precisely as element of the factor space $\mathcal{G}_{\mathcal{F} \mathcal{L}, a}\left(\mathbb{C}^{n}\right)$ (see [11, Subsection 2.3]).

Proposition 2. For all $M \in \mathbb{N}$ and $a>0$ there exists a constant $c_{M, a}>0$ such that the inequality

$$
|\mathcal{F} \mathcal{L}(u)(\zeta)| \leq c_{M, a}(1+|\zeta|)^{-M} \sup _{|\alpha| \leq M,|x| \leq a}\left|\partial^{\alpha} u(x)\right| \sup _{|x| \leq a} \mathrm{e}^{x \operatorname{Im}(\zeta)}
$$

holds for all $\zeta \in \mathbb{C}^{n}$ and for all $u \in \mathcal{C}^{\infty}\left(\mathbb{R}^{n}\right)$ with $\operatorname{supp} u \subseteq\{x:|x| \leq a\}$.

2.2 Partial differential operators with constant Colombeau coefficients: generalized polynomials and fundamental solutions. Let

$$
P(D)=\sum_{|\alpha| \leq m} c_{\alpha} D^{\alpha}
$$

be a partial differential operator of order $m$ with coefficients in $\widetilde{\mathbb{C}}$. To $P(D)$ we associate the generalized polynomial

$$
P(\xi)=\sum_{|\alpha| \leq m} c_{\alpha} \xi^{\alpha}
$$


Any net of polynomials $\left(P_{\varepsilon}\right)_{\varepsilon}$ determined by a choice of representatives of the coefficients of $P$ is called a representative of $P$. Consider the function $\widetilde{P}: \mathbb{R}^{n} \rightarrow \widetilde{\mathbb{R}}$ defined by

$$
\widetilde{P}^{2}(\xi)=\sum_{|\alpha| \leq m}\left|\partial^{\alpha} P(\xi)\right|^{2}
$$

The arguments in [16, Example 10.1.3] yield the following assertion: there exists $C>0$ depending only on $m$ and $n$ such that for all $\left(P_{\varepsilon}\right)_{\varepsilon}$ the inequality

$$
\widetilde{P_{\varepsilon}}(\xi+\eta) \leq(1+C|\xi|)^{m} \widetilde{P_{\varepsilon}}(\eta)
$$

is valid for all $\xi, \eta \in \mathbb{R}^{n}$ and all $\varepsilon \in(0,1]$. When the function $\widetilde{P}: \mathbb{R}^{n} \rightarrow \widetilde{\mathbb{R}}$ is invertible in some point $\xi_{0}$ of $\mathbb{R}^{n}$ Lemma 7.5 in [17] proves that for all representative $\left(P_{\varepsilon}\right)_{\varepsilon}$ of $P$ there exist $N \in \mathbb{N}$ and $\eta \in(0,1]$ such that

$$
\widetilde{P_{\varepsilon}}(\xi) \geq \varepsilon^{N}\left(1+C\left|\xi_{0}-\xi\right|\right)^{-m},
$$

for all $\xi \in \mathbb{R}^{n}$ and $\varepsilon \in(0, \eta]$. Note that the constant $C>0$ is the same appearing in (4) and $\varepsilon^{N}$ comes from the invertibility in $\widetilde{\mathbb{R}}$ of $\widetilde{P}\left(\xi_{0}\right)$.

The operator $P(D)$ maps $\mathcal{G}_{\mathrm{c}}(\Omega), \mathcal{G}_{\mathrm{c}}^{\infty}(\Omega), \mathcal{G}(\Omega), \mathcal{G}^{\infty}(\Omega), \mathcal{L}(\mathcal{G}(\Omega), \widetilde{\mathbb{C}}), \mathcal{L}_{\mathrm{b}}(\mathcal{G}(\Omega), \widetilde{\mathbb{C}})$, $\mathcal{L}\left(\mathcal{G}_{\mathrm{c}}(\Omega), \widetilde{\mathbb{C}}\right)$ and $\mathcal{L}_{\mathrm{b}}\left(\mathcal{G}_{\mathrm{c}}(\Omega), \widetilde{\mathbb{C}}\right)$ into themselves respectively for any open subset $\Omega$ of $\mathbb{R}^{n}$. Working at the level of representatives one can easily prove that

$$
P(D)(S * T)=P(D) S * T=S * P(D) T
$$

holds for all $S \in \mathcal{L}\left(\mathcal{G}\left(\mathbb{R}^{n}\right), \widetilde{\mathbb{C}}\right)$ and $T \in \mathcal{L}_{\mathrm{b}}\left(\mathcal{G}_{\mathrm{c}}\left(\mathbb{R}^{n}\right), \widetilde{\mathbb{C}}\right)$.

The notion of fundamental solution of $P(D)$ is introduced in [11, Definition 3.1] within the dual of the Colombeau algebra $\mathcal{G}_{\mathrm{c}}\left(\mathbb{R}^{n}\right)$. In detail, we say that $E \in \mathcal{L}\left(\mathcal{G}_{\mathrm{c}}\left(\mathbb{R}^{n}\right), \widetilde{\mathbb{C}}\right)$ is a fundamental solution of $P(D)$ if

$$
P(D) E=\iota_{d}(\delta)
$$

in $\mathcal{L}\left(\mathcal{G}_{\mathrm{c}}\left(\mathbb{R}^{n}\right), \widetilde{\mathbb{C}}\right)$. When the function $\widetilde{P}$ associated to the operator $P(D)$ is invertible in some point of $\mathbb{R}^{n}$, Theorem 3.3 in [11] proves that $P(D)$ admits a fundamental solution in $\mathcal{L}_{\mathrm{b}}\left(\mathcal{G}_{\mathrm{c}}\left(\mathbb{R}^{n}\right), \widetilde{\mathbb{C}}\right)$. This is the Malgrange-Ehrenpreiss Theorem for fundamental solutions in the space $\mathcal{L}_{\mathrm{b}}\left(\mathcal{G}_{\mathrm{c}}\left(\mathbb{R}^{n}\right), \widetilde{\mathbb{C}}\right)$.

3. General approach to $\mathcal{G}$ - and $\mathcal{G}^{\infty}$-hypoellipticity Before dealing with partial differential operators with $\widetilde{\mathbb{C}}$-coefficients, we approach the general problem of hypoellipticity in the wider context of continuous $\widetilde{\mathbb{C}}$-linear maps acting from $\mathcal{G}_{\mathrm{c}}\left(\Omega^{\prime}\right)$ to $\mathcal{L}\left(\mathcal{G}_{\mathrm{c}}(\Omega), \widetilde{\mathbb{C}}\right)$, where $\Omega^{\prime}$ and $\Omega$ are open subsets of $\mathbb{R}^{n^{\prime}}$ and $\mathbb{R}^{n}$ respectively. The results of this section are inspired by $\left[21\right.$, Section 2.1]. Note that if $k: \mathcal{G}_{\mathrm{c}}\left(\Omega^{\prime}\right) \rightarrow \mathcal{L}\left(\mathcal{G}_{\mathrm{c}}(\Omega), \widetilde{\mathbb{C}}\right)$ is a continuous $\widetilde{\mathbb{C}}$-linear map fulfilling the following condition

$$
\forall K^{\prime} \Subset \Omega^{\prime}, \forall K \Subset \Omega, \exists c>0 \exists j \in \mathbb{N} \forall v \in \mathcal{G}_{K^{\prime}}\left(\Omega^{\prime}\right) \forall u \in \mathcal{G}_{K}(\Omega),
$$

$$
|k(v)(u)|_{\mathrm{e}} \leq c \mathcal{P}_{\mathcal{G}_{K^{\prime}}\left(\Omega^{\prime}\right), j}(v) \mathcal{P}_{\mathcal{G}_{K}(\Omega), j}(u),
$$

then the formula

$$
{ }^{t} k(u)(v)=k(v)(u)
$$

defines a continuous $\widetilde{\mathbb{C}}$-linear map from $\mathcal{G}_{\mathrm{c}}(\Omega)$ to $\mathcal{L}\left(\mathcal{G}_{\mathrm{c}}\left(\Omega^{\prime}\right), \widetilde{\mathbb{C}}\right)$. Throughout this section, every time we deal with a map $k: \mathcal{G}_{\mathrm{c}}\left(\Omega^{\prime}\right) \rightarrow \mathcal{L}\left(\mathcal{G}_{\mathrm{c}}(\Omega), \widetilde{\mathbb{C}}\right)$ we assume the continuity 
property (6).

DEFinition 3.

Let $\Omega \subseteq \mathbb{R}_{x}^{n}$ and $\Omega^{\prime} \subseteq \mathbb{R}_{y}^{n^{\prime}}$ be open subsets. Let

$$
k: \mathcal{G}_{\mathrm{c}}\left(\Omega^{\prime}\right) \rightarrow \mathcal{L}\left(\mathcal{G}_{\mathrm{c}}(\Omega), \widetilde{\mathbb{C}}\right)
$$

be a $\widetilde{\mathbb{C}}$-linear continuous map satisfying condition $(6) . k$ is said to be

(i) $\mathcal{G}$-semi-regular in $x$ if it maps $\mathcal{G}_{\mathrm{c}}\left(\Omega^{\prime}\right)$ continuously in $\mathcal{G}(\Omega)$;

(ii) $\mathcal{G}^{\infty}$-semi-regular in $x$ if it maps $\mathcal{G}_{\mathrm{c}}^{\infty}\left(\Omega^{\prime}\right)$ continuously in $\mathcal{G}^{\infty}(\Omega)$;

(iii) $\mathcal{G}$-semi-regular in $y$ if ${ }^{t} k$ maps $\mathcal{G}_{\mathrm{c}}(\Omega)$ continuously in $\mathcal{G}\left(\Omega^{\prime}\right)$;

(iv) $\mathcal{G}^{\infty}$-semi-regular in $y$ if ${ }^{t} k$ maps $\mathcal{G}_{\mathrm{c}}^{\infty}(\Omega)$ continuously in $\mathcal{G}^{\infty}\left(\Omega^{\prime}\right)$;

(v) $\mathcal{G}$-regular if it is $\mathcal{G}$-semi-regular in $x$ and $y$;

(vi) $\mathcal{G}^{\infty}$-regular if it is $\mathcal{G}^{\infty}$-semi-regular in $x$ and $y$.

If $n=n^{\prime}$ and $\Omega^{\prime}=\Omega$ then $k$ is said to be $\mathcal{G}$-very regular if it is $\mathcal{G}$-regular and there exists $w \in \mathcal{G}(\Omega \times \Omega \backslash \Delta)$ such that

$$
k(u)(v)=\int w(x, y) u(y) v(x) d x d y
$$

for all $u, v \in \mathcal{G}_{\mathrm{c}}(\Omega)$ with $\operatorname{supp} u \cap \operatorname{supp} v=\emptyset$. Analogously, $k$ is $\mathcal{G}^{\infty}$-very regular if it is $\mathcal{G}^{\infty}$-regular and (7) holds with $w \in \mathcal{G}^{\infty}(\Omega \times \Omega \backslash \Delta)$. Note that if $k$ is $\mathcal{G}$-semi-regular in $y$ then it extends to a continuous $\widetilde{\mathbb{C}}$-linear map from $\mathcal{L}\left(\mathcal{G}\left(\Omega^{\prime}\right), \widetilde{\mathbb{C}}\right)$ to $\mathcal{L}\left(\mathcal{G}_{\mathrm{c}}(\Omega), \widetilde{\mathbb{C}}\right)$. This extension is given by $k(T)(u)=T\left({ }^{t} k(u)\right)$ for $T \in \mathcal{L}\left(\mathcal{G}\left(\Omega^{\prime}\right), \widetilde{\mathbb{C}}\right)$ and $u \in \mathcal{G}_{\mathrm{c}}(\Omega)$.

REMARK 1. The kernel of a generalized pseudodifferential operator can be associated to a map as in Definition 3. In detail, let $K_{a(x, D)} \in \mathcal{L}\left(\mathcal{G}_{\mathrm{c}}(\Omega \times \Omega)\right.$, $\left.\widetilde{\mathbb{C}}\right)$ be the kernel of the pseudodifferential operator $a(x, D)$ with $a \in \widetilde{\mathcal{S}}_{\rho, \delta}^{m}\left(\Omega \times \mathbb{R}^{n}\right)$ (for the definition of $\widetilde{\mathcal{S}}_{\rho, \delta}^{m}\left(\Omega \times \mathbb{R}^{n}\right)$ and the theory of pseudodifferential operators acting on Colombeau algebras, see $[9,12]) \cdot K_{a(x, D)}$ defines the map

$$
k_{a}: \mathcal{G}_{\mathrm{c}}(\Omega) \rightarrow \mathcal{L}\left(\mathcal{G}_{\mathrm{c}}(\Omega), \widetilde{\mathbb{C}}\right)
$$

as follows:

$$
k_{a}(u)(v)=K_{a(x, D)}(v \otimes u)=\int_{\mathbb{R}^{n}} a(x, D) u(x) v(x) d x .
$$

From Propositions 2.1 and 2.8 in [9] we have that $k_{a}$ is $\mathcal{G}$-very-regular and in particular $\mathcal{G}^{\infty}$-very regular when $a$ belongs to the smaller set $\widetilde{\mathcal{S}}_{\rho, \delta, \mathrm{rg}}^{m}\left(\Omega \times \mathbb{R}^{n}\right) \subseteq \widetilde{\mathcal{S}}_{\rho, \delta}^{m}\left(\Omega \times \mathbb{R}^{n}\right)$ of $\left(\mathcal{G}^{\infty}\right.$-)regular symbols as defined in [Section 2, 9]. It follows that any partial differential operator $P(D)$ with generalized constant coefficients can be regarded as a map $k_{P}$ which is both $\mathcal{G}$ - and $\mathcal{G}^{\infty}$-very regular.

Definition 4.

Let $k: \mathcal{G}_{\mathrm{c}}(\Omega) \rightarrow \mathcal{L}\left(\mathcal{G}_{\mathrm{c}}(\Omega), \widetilde{\mathbb{C}}\right)$ be $\mathcal{G}$-semi-regular in $y$. We say that $k$ has 
(i) the $\mathcal{G}$-pseudolocal property if

$$
\operatorname{sing} \operatorname{supp}_{\mathcal{G}} k(T) \subseteq \operatorname{sing} \operatorname{supp}_{\mathcal{G}} T
$$

for all $T \in \mathcal{L}_{\mathrm{b}}(\mathcal{G}(\Omega), \widetilde{\mathbb{C}}) ;$

(ii) the $\mathcal{G}^{\infty}$-pseudolocal property if

$$
\operatorname{sing} \operatorname{supp}_{\mathcal{G}_{\infty}} k(T) \subseteq \operatorname{sing} \operatorname{supp}_{\mathcal{G} \infty} T
$$

for all $T \in \mathcal{L}_{\mathrm{b}}(\mathcal{G}(\Omega), \widetilde{\mathbb{C}})$

Proposition 5. If $k$ is $\mathcal{G}$-very regular then it has the $\mathcal{G}$-pseudolocal property. If $k$ is $\mathcal{G}^{\infty}$-very regular then it has the $\mathcal{G}^{\infty}$-pseudolocal property

Proof. Let $T \in \mathcal{L}_{\mathrm{b}}(\mathcal{G}(\Omega), \widetilde{\mathbb{C}})$ and $x_{0} \in\left(\operatorname{sing} \operatorname{supp}_{\mathcal{G}} T\right)^{\mathrm{c}}$. We can take a cut-off function $\psi \in \mathcal{C}_{\mathrm{c}}^{\infty}(\Omega)$ identically 1 in a neighborhood $V$ of $x_{0}$ such that $\psi T \in \mathcal{G}_{\mathrm{c}}(\Omega)$. We write $k(T)$ as $k(\psi T)+k((1-\psi) T)$. Since $k$ is $\mathcal{G}$-very regular we have $k(\psi T) \in \mathcal{G}(\Omega)$. Let $\varphi \in \mathcal{C}_{\mathrm{c}}^{\infty}(\Omega)$ be identically 1 in neighborhood of $\operatorname{supp} T$ and $u \in \mathcal{G}_{\mathrm{c}}(V)$. We have that $k((1-\psi) T)(u)$ coincides with

$$
k((1-\psi) \varphi T)(u)=(1-\psi) \varphi T\left({ }^{t} k u\right)=T\left((1-\psi) \varphi^{t} k(u)\right) .
$$

The assumption of $\mathcal{G}$-very regularity on $k$ and the fact that $(1-\psi) \varphi$ and $u$ have supports disjoint yield

$$
k((1-\psi) T)(u)=T_{y}\left(\int w(x, y)(1-\psi)(y) \varphi(y) u(x) d x\right),
$$

where $\int w(x, y)(1-\psi)(y) \varphi(y) u(x) d x \in \mathcal{G}\left(\mathbb{R}^{n}\right)$. Since $T$ is a basic functional, a combination of Propositions 1.4, 1.8 and 1.9 of [9] allows to conclude that

$$
k((1-\psi) T)(u)=\int_{\mathbb{R}^{n}} T(w(x, \cdot)(1-\psi)(\cdot) \varphi(\cdot)) u(x) d x,
$$

with $T(w(x, \cdot)(1-\psi)(\cdot) \varphi(\cdot)) \in \mathcal{G}(V)$. It follows that $x_{0} \in\left(\operatorname{sing} \operatorname{supp}_{\mathcal{G}} k(T)\right)^{\mathrm{c}}$. When $k$ is $\mathcal{G}^{\infty}$-regular, one repeats the previous arguments with $\mathcal{G}^{\infty}$ in place of $\mathcal{G}$.

Definition 6.

The continuous $\widetilde{\mathbb{C}}$-linear map $k: \mathcal{G}_{\mathrm{c}}(\Omega) \rightarrow \mathcal{L}\left(\mathcal{G}_{\mathrm{c}}(\Omega), \widetilde{\mathbb{C}}\right)$ is said to be

(i) properly supported if

$$
\text { for all } K \Subset \Omega \text { there exist } K^{\prime} \Subset \Omega \text { and } K^{\prime \prime} \Subset \Omega \text { such that }
$$

- $\operatorname{supp} u \subseteq K \Rightarrow \operatorname{supp} k(u) \subseteq K^{\prime}$,

- $\operatorname{supp} u \subseteq K \Rightarrow \operatorname{supp}^{t} k(u) \subseteq K^{\prime \prime}$,

- $\operatorname{supp} u \subseteq \Omega \backslash K^{\prime \prime} \Rightarrow \operatorname{supp} k(u) \subseteq \Omega \backslash K$,

- $\operatorname{supp} u \subseteq \Omega \backslash K^{\prime} \Rightarrow \operatorname{supp}^{t} k(u) \subseteq \Omega \backslash K$;

(ii) $\mathcal{G}$-regularizing if it is $\mathcal{G}$-semi-regular in $y$ and maps $\mathcal{L}(\mathcal{G}(\Omega), \widetilde{\mathbb{C}})$ into $\mathcal{G}(\Omega)$ continuously;

(iii) $\mathcal{G}^{\infty}$-regularizing if it is $\mathcal{G}$-semi-regular in $y$ and maps $\mathcal{L}(\mathcal{G}(\Omega), \widetilde{\mathbb{C}})$ into $\mathcal{G}^{\infty}(\Omega)$ continuously. 
If $k$ is properly supported then it maps $\mathcal{G}_{\mathrm{c}}(\Omega)$ to $\mathcal{L}(\mathcal{G}(\Omega), \widetilde{\mathbb{C}})$ and it extends to a continuous $\widetilde{\mathbb{C}}$-linear map from $\mathcal{G}(\Omega)$ to $\mathcal{L}\left(\mathcal{G}_{\mathrm{c}}(\Omega), \widetilde{\mathbb{C}}\right)$. Indeed, let $V_{1} \subset V_{2} \subset \ldots$ be an exhausting sequence of relatively compact subsets of $\Omega, K_{j}=\overline{V_{j}}$ and $K_{j}^{\prime \prime}$ as in Definition $6(\mathrm{i})$. Taking $\psi_{j} \in \mathcal{C}_{\mathrm{c}}^{\infty}(\Omega)$ identically one in a neighborhood of $K_{j}^{\prime \prime}$, for any $u \in \mathcal{G}(\Omega)$ we have that $k_{j} u=\left.k\left(\psi_{j} u\right)\right|_{V_{j}}$ defines a coherent family of elements of $\mathcal{L}\left(\mathcal{G}_{\mathrm{c}}(\Omega), \widetilde{\mathbb{C}}\right)$. In this way we conclude that $k$ can be uniquely extended to a map from $\mathcal{G}(\Omega)$ to $\mathcal{L}\left(\mathcal{G}_{\mathrm{c}}(\Omega), \widetilde{\mathbb{C}}\right)$ such that

$$
k(u)(v)={ }^{t} k(v)(u)
$$

for all $u \in \mathcal{G}(\Omega)$ and $v \in \mathcal{G}_{\mathrm{c}}(\Omega)$. In particular, a properly supported $\mathcal{G}$-regular map $k$ has the mapping properties $\mathcal{G}_{\mathrm{c}}(\Omega) \rightarrow \mathcal{G}_{\mathrm{c}}(\Omega), \mathcal{G}(\Omega) \rightarrow \mathcal{G}(\Omega), \mathcal{L}(\mathcal{G}(\Omega), \widetilde{\mathbb{C}}) \rightarrow \mathcal{L}(\mathcal{G}(\Omega), \widetilde{\mathbb{C}})$, $\mathcal{L}\left(\mathcal{G}_{\mathrm{c}}(\Omega), \widetilde{\mathbb{C}}\right) \rightarrow \mathcal{L}\left(\mathcal{G}_{\mathrm{c}}(\Omega), \widetilde{\mathbb{C}}\right)$. Replacing $\mathcal{G}$ with $\mathcal{G}^{\infty}$ we obtain the mapping properties of a properly supported $\mathcal{G}^{\infty}$-regular map. Moreover, if $k$ is $\mathcal{G}$-regularizing and properly supported then it maps $\mathcal{L}(\mathcal{G}(\Omega), \widetilde{\mathbb{C}})$ into $\mathcal{G}_{\mathrm{c}}(\Omega)$ and $\mathcal{L}\left(\mathcal{G}_{\mathrm{c}}(\Omega), \widetilde{\mathbb{C}}\right)$ into $\mathcal{G}(\Omega)$. Analogously, if it is $\mathcal{G}^{\infty}$-regularizing and properly supported then it maps the duals $\mathcal{L}(\mathcal{G}(\Omega), \widetilde{\mathbb{C}})$ and $\mathcal{L}\left(\mathcal{G}_{\mathrm{c}}(\Omega), \widetilde{\mathbb{C}}\right)$ into $\mathcal{G}_{\mathrm{c}}^{\infty}(\Omega)$ and $\mathcal{G}^{\infty}(\Omega)$ respectively.

\section{Definition 7.}

Let $k$ be a $\mathcal{G}$-very regular map. We say that the $\mathcal{G}$-regular properly supported map $k^{\prime}$ is a left $\mathcal{G}$-parametrix of $k$ if there exists a $\mathcal{G}$-regularizing map $r$ such that

$$
k^{\prime} k(T)-r(T)=T
$$

in $\mathcal{L}\left(\mathcal{G}_{\mathrm{c}}(\Omega), \widetilde{\mathbb{C}}\right)$ for all $T \in \mathcal{L}_{\mathrm{b}}(\mathcal{G}(\Omega), \widetilde{\mathbb{C}})$.

Let $k$ be a $\mathcal{G}^{\infty}$-very regular map. We say that the $\mathcal{G}^{\infty}$-regular properly supported map $k^{\prime}$ is a left $\mathcal{G}^{\infty}$-parametrix of $k$ if if there exists a $\mathcal{G}^{\infty}$-regularizing map $r$ such that (8) holds for all $T \in \mathcal{L}_{\mathrm{b}}(\mathcal{G}(\Omega), \widetilde{\mathbb{C}})$.

If $k$ is properly supported then we omit the assumption of properly supported map in the definition of $\mathcal{G}$ - and $\mathcal{G}^{\infty}$-parametrix. When $r=0$ then $k$ has a $\mathcal{G}$-regular or $\mathcal{G}^{\infty}$-regular left inverse. Clearly one can define a right $\mathcal{G}$ - or $\mathcal{G}^{\infty}$-parametrix $k$ substituting $k^{\prime} k$ with $k k^{\prime}$ in (8).

\section{TheOREM 8.}

(i) If the $\mathcal{G}$-very regular map $k$ has a $\mathcal{G}$-very regular left parametrix map then

$$
\operatorname{sing} \operatorname{supp}_{\mathcal{G}} k(T)=\operatorname{sing} \operatorname{supp}_{\mathcal{G}} T
$$

for all $T \in \mathcal{L}_{\mathrm{b}}(\mathcal{G}(\Omega), \widetilde{\mathbb{C}})$.

(ii) If the $\mathcal{G}^{\infty}$-very regular map $k$ has a $\mathcal{G}^{\infty}$-very regular left parametrix map then

$$
\text { for all } T \in \mathcal{L}_{\mathrm{b}}(\mathcal{G}(\Omega), \widetilde{\mathbb{C}}) .
$$

Proof. From Proposition 5 we already know that $\operatorname{sing} \operatorname{supp}_{\mathcal{G}} k(T) \subseteq \operatorname{sing} \operatorname{supp}_{\mathcal{G}} T$ for all $T \in \mathcal{L}_{\mathrm{b}}(\mathcal{G}(\Omega), \widetilde{\mathbb{C}})$. Since sing $\operatorname{supp}_{\mathcal{G}} T=\operatorname{sing} \operatorname{supp}_{\mathcal{G}} k^{\prime} k(T)-r(T)$ and $r$ is $\mathcal{G}$-regularizing we have $\operatorname{sing} \operatorname{supp}_{\mathcal{G}} T=\operatorname{sing} \operatorname{supp}_{\mathcal{G}} k^{\prime} k(T) \subseteq \operatorname{sing} \operatorname{supp}_{\mathcal{G}} k(T)$. The $\mathcal{G}^{\infty}$-case is analogous. 
We finally concentrate on partial differential operators $P(D)$ with constant Colombeau coefficients, regarded as $\mathcal{G}$ - and $\mathcal{G}^{\infty}$-very regular maps. We begin by observing that the $\mathcal{G}$ - and $\mathcal{G}^{\infty}$-pseudolocality property are valid for all functionals $T \in \mathcal{L}_{\mathrm{b}}\left(\mathcal{G}_{\mathrm{c}}\left(\mathbb{R}^{n}\right), \widetilde{\mathbb{C}}\right)$. This follows from the fact that for all relatively compact open subsets $\Omega$ of $\mathbb{R}^{n}$,

$$
\left.(P(D) T)\right|_{\Omega}=P(D)\left(\left.T\right|_{\Omega}\right)=\left.P(D)(\psi T)\right|_{\Omega},
$$

where $\psi \in \mathcal{C}_{\mathrm{c}}^{\infty}\left(\mathbb{R}^{n}\right)$ is identically 1 on $\bar{\Omega}$. Hence,

$$
\operatorname{sing} \operatorname{supp}_{\mathcal{G}} P(D) T \subseteq \operatorname{sing} \operatorname{supp}_{\mathcal{G}} T
$$

and

$$
\text { sing } \operatorname{supp}_{\mathcal{G} \infty} P(D) T \subseteq \operatorname{sing} \operatorname{supp}_{\mathcal{G}} T
$$

for all $T \in \mathcal{L}_{\mathrm{b}}\left(\mathcal{G}_{\mathrm{c}}\left(\mathbb{R}^{n}\right), \widetilde{\mathbb{C}}\right)$.

In the next proposition we prove that a certain class of partial differential operators with constant Colombeau coefficients admit a very regular inverse, i.e., a very regular left and right inverse. This result will be employed in Section 4 in order to characterize the $\mathcal{G}$ - and $\mathcal{G}^{\infty}$-hypoellipticity.

Proposition 9. Let $P(D)$ be a partial differential operator with $\widetilde{\mathbb{C}}$-coefficients.

(i) If $P(D)$ has a fundamental solution $E \in \mathcal{L}_{\mathrm{b}}\left(\mathcal{G}_{\mathrm{c}}\left(\mathbb{R}^{n}\right)\right.$, $\left.\widetilde{\mathbb{C}}\right)$ which belongs to $\mathcal{G}$ outside the origin then it admits a $\mathcal{G}$-very regular inverse map.

(ii) If $P(D)$ has a fundamental solution $E \in \mathcal{L}_{\mathrm{b}}\left(\mathcal{G}_{\mathrm{c}}\left(\mathbb{R}^{n}\right)\right.$, $\left.\widetilde{\mathbb{C}}\right)$ which belongs to $\mathcal{G}^{\infty}$ outside the origin then it admits a $\mathcal{G}^{\infty}$-very regular inverse map.

Proof. (i) Let us define the map

$$
k^{\prime}: \mathcal{G}_{\mathrm{c}}\left(\mathbb{R}^{n}\right) \rightarrow \mathcal{L}\left(\mathcal{G}_{\mathrm{c}}\left(\mathbb{R}^{n}\right), \widetilde{\mathbb{C}}\right): u \rightarrow u * E .
$$

From the properties of the convolution between a basic functional and a Colombeau generalized function (see Propositions 1.9 and 1.12 in [9] and Proposition 1) we have that $k^{\prime}$ is both a $\mathcal{G}$ - and a $\mathcal{G}^{\infty}$-regular map. Since $E$ belongs to $\mathcal{G}$ outside the origin we can write, for $u, v \in \mathcal{G}_{\mathrm{c}}\left(\mathbb{R}^{n}\right)$ with $\operatorname{supp} u \cap \operatorname{supp} v=\emptyset$,

$$
k^{\prime}(u)(v)=\int_{\mathbb{R}^{n}} E(x-y) u(y) v(x) d x d y,
$$

where $E(x-y) \in \mathcal{G}\left(\mathbb{R}^{2 n} \backslash \Delta\right)$. Hence, $k^{\prime}$ is $\mathcal{G}$-very regular. Under the hypothesis of $(i i)$ we have that $E(x-y) \in \mathcal{G}^{\infty}\left(\mathbb{R}^{2 n} \backslash \Delta\right)$ and therefore $k^{\prime}$ is $\mathcal{G}^{\infty}$-very regular. Concluding, setting $k=P(D)$ from (5) we obtain

$$
k^{\prime} k(T)=P(D) T * E=T * P(D) E=T
$$

and

$$
k k^{\prime}(T)=P(D)(T * E)=T * P(D) E=T
$$

valid for all $T \in \mathcal{L}_{\mathrm{b}}\left(\mathcal{G}_{\mathrm{c}}\left(\mathbb{R}^{n}\right), \widetilde{\mathbb{C}}\right)$. 
4. $\mathcal{G}$ - and $\mathcal{G}^{\infty}$-hypoellipticity: characterizations and necessary conditions. In this section we introduce the notion of $\mathcal{G}$ - and $\mathcal{G}^{\infty}$-hypoellipticity for operators with constant Colombeau coefficients. The characterizations and necessary conditions here collected are the main topic of Section 4 in [11], to which we address the reader for detailed proofs and further explanations.

\section{Definition 10.}

Let $P(D)$ be a partial differential operator with coefficients in $\widetilde{\mathbb{C}} . P(D)$ is said to be $\mathcal{G}$-hypoelliptic if for any open subset $\Omega$ of $\mathbb{R}^{n}$ the equality

$$
\text { sing } \operatorname{supp}_{\mathcal{G}} P(D) T=\operatorname{sing} \operatorname{supp}_{\mathcal{G}} T
$$

holds for all basic functionals $T \in \mathcal{L}\left(\mathcal{G}_{\mathrm{c}}(\Omega), \widetilde{\mathbb{C}}\right)$. Analogously, $P(D)$ is said to be $\mathcal{G}^{\infty}$ hypoelliptic if for any open subset $\Omega$ of $\mathbb{R}^{n}$,

$$
\operatorname{sing} \operatorname{supp}_{\mathcal{G}^{\infty}} P(D) T=\operatorname{sing} \operatorname{supp}_{\mathcal{G}} \infty T
$$

for all basic functionals $T \in \mathcal{L}\left(\mathcal{G}_{\mathrm{c}}(\Omega), \widetilde{\mathbb{C}}\right)$.

Definition 10 can be equivalently stated by requiring that (11) and (12) are valid for all basic functionals of $\mathcal{L}\left(\mathcal{G}_{\mathrm{c}}\left(\mathbb{R}^{n}\right), \widetilde{\mathbb{C}}\right)$. From the pseudolocality property of $P(D)$ it follows that $P(D)$ is $\mathcal{G}$-hypoelliptic if and only if for all open subsets $X$ of $\mathbb{R}^{n}$ and all basic functionals $T$ of $\mathcal{L}\left(\mathcal{G}_{\mathrm{c}}\left(\mathbb{R}^{n}\right), \widetilde{\mathbb{C}}\right),\left.P(D) T\right|_{X} \in \mathcal{G}(X)$ implies $\left.T\right|_{X} \in \mathcal{G}(X)$. Analogously, replacing $\mathcal{G}$ with $\mathcal{G}^{\infty}$ we obtain another equivalent formulation of the $\mathcal{G}^{\infty}$-hypoellipticity of $P(D)$. As observed in the previous section, it is not restrictive to take $T \in \mathcal{L}_{\mathrm{b}}\left(\mathcal{G}\left(\mathbb{R}^{n}\right), \widetilde{\mathbb{C}}\right)$ in (11) and (12).

For operators with constant Colombeau coefficients the $\mathcal{G}$-hypoellipticity as well as the $\mathcal{G}^{\infty}$-hypoellipticity may be characterized making use of the fundamental solutions. This requires the following definition.

\section{Definition 11.}

We say that $F \in \mathcal{L}_{\mathrm{b}}\left(\mathcal{G}_{\mathrm{c}}\left(\mathbb{R}^{n}\right), \widetilde{\mathbb{C}}\right)$ is a $\mathcal{G}$-parametrix of $P(D)$ if

$$
P(D) F-\iota_{d}(\delta) \in \mathcal{G}\left(\mathbb{R}^{n}\right)
$$

and that $F \in \mathcal{L}_{\mathrm{b}}\left(\mathcal{G}_{\mathrm{c}}\left(\mathbb{R}^{n}\right), \widetilde{\mathbb{C}}\right)$ is a $\mathcal{G}^{\infty}$-parametrix of $P(D)$ if

$$
P(D) F-\iota_{d}(\delta) \in \mathcal{G}^{\infty}\left(\mathbb{R}^{n}\right) .
$$

In the sequel, we assume that the function $\widetilde{P}$ associated to the operator $P(D)$ is invertible in some point of $\mathbb{R}^{n}$ and hence we know that the operator $P(D)$ admits a fundamental solution in $\mathcal{L}_{\mathrm{b}}\left(\mathcal{G}_{\mathrm{c}}\left(\mathbb{R}^{n}\right), \widetilde{\mathbb{C}}\right)$. We are now ready to state the following characterization of generalized hypoellipticity [11, Theorem 4.2].

Theorem 12. Let $P(D)$ be a partial differential operator with constant Colombeau coefficients such that the function $\widetilde{P}$ is invertible in some point of $\mathbb{R}^{n}$. The following assertions are equivalent:

(i) the operator $P(D)$ is $\mathcal{G}$-hypoelliptic in $\mathbb{R}^{n}$,

(ii) the operator $P(D)$ has a fundamental solution $E \in \mathcal{L}_{\mathrm{b}}\left(\mathcal{G}_{\mathrm{c}}\left(\mathbb{R}^{n}\right), \widetilde{\mathbb{C}}\right)$ with $\operatorname{sing} \operatorname{supp}_{\mathcal{G}} E$ $\subseteq\{0\}$ 
(iii) the operator $P(D)$ has a $\mathcal{G}$-parametrix $F \in \mathcal{L}_{\mathrm{b}}\left(\mathcal{G}_{\mathrm{c}}\left(\mathbb{R}^{n}\right), \widetilde{\mathbb{C}}\right)$ with $\operatorname{sing} \operatorname{supp}_{\mathcal{G}} F \subseteq\{0\}$.

The same kind of equivalence holds with $\mathcal{G}^{\infty}$-hypoelliptic, $\operatorname{sing} \operatorname{supp}_{\mathcal{G}^{\infty}}$ and $\mathcal{G}^{\infty}$-parametrix in place of $\mathcal{G}$-hypoelliptic, $\operatorname{sing} \operatorname{supp}_{\mathcal{G}}$ and $\mathcal{G}$-parametrix respectively.

Combining the previous theorem with Proposition 9 we deduce this other characterization of $\mathcal{G}$ - and $\mathcal{G}^{\infty}$-hypoellpticity where the inverse map are intended on $\mathcal{L}_{\mathrm{b}}\left(\mathcal{G}\left(\mathbb{R}^{n}\right), \widetilde{\mathbb{C}}\right)$.

Corollary 13. Let $P(D)$ be a partial differential operator with constant Colombeau coefficients such that the function $\widetilde{P}$ is invertible in some point of $\mathbb{R}^{n}$.

(i) $P(D)$ is $\mathcal{G}$-hypoellptic if and only if it admits a $\mathcal{G}$-very regular inverse map;

(ii) $P(D)$ is $\mathcal{G}^{\infty}$-hypoelliptic if and only if it admits a $\mathcal{G}^{\infty}$-very regular inverse map.

The recent investigation of the $\mathcal{G}$ - and $\mathcal{G}^{\infty}$-regularity properties of generalized differential and pseudodifferential operators in the Colombeau context $[6,12,13,17,18]$ has provided several sufficient conditions of $\mathcal{G}$ - and $\mathcal{G}^{\infty}$-hypoellipticity. The search for necessary conditions for $\mathcal{G}$ - and $\mathcal{G}^{\infty}$-hypoellipticity has been a long-standing open problem. The first necessary condition for $\mathcal{G}^{\infty}$-hypoellipticity has be obtained by the author in [10] by making use of a generalized version of the closed graph theorem valid for Fréchet $\widetilde{\mathbb{C}}$-modules. Since this is also one of the first applications of functional analysis in the Colombeau context, we give a detailed proof of Theorem 14 .

Before going on, we recall, that $\xi \in \widetilde{\mathbb{R}}^{n}$ is of log-type if there exists a representative $\left(\xi_{\varepsilon}\right)_{\varepsilon}$ of $\xi \in \widetilde{\mathbb{R}}^{n}$ such that $\left|\xi_{\varepsilon}\right|=O(\log (1 / \varepsilon))$. When $\xi$ is of log-type the generalized number

$$
\mathrm{e}^{|\xi|}:=\left[\left(\mathrm{e}^{\left|\xi_{\varepsilon}\right|}\right)_{\varepsilon}\right],
$$

where $\left(\xi_{\varepsilon}\right)_{\varepsilon}$ is any representative of $\xi$ such that $\left(\mathrm{e}^{\left|\xi_{\varepsilon}\right|}\right)_{\varepsilon}$ is moderate, is well-defined in $\widetilde{\mathbb{R}}$. Analogously, if $\zeta \in \widetilde{\mathbb{C}}^{n}$ and $\operatorname{Im} \zeta$ is of log-type then $\mathrm{e}^{|\operatorname{Im} \zeta|} \in \widetilde{\mathbb{R}}$. Moreover, when $\zeta \in \widetilde{\mathbb{C}}^{n}$ and $\operatorname{Im} \zeta$ is of log-type then

$$
\mathrm{e}^{-i x \zeta}:=\left[\left(\mathrm{e}^{-i x \zeta_{\varepsilon}}\right)_{\varepsilon}\right]
$$

is a generalized function in $\mathcal{G}\left(\mathbb{R}^{n}\right)$.

THEOREM 14. Let $P(D)$ be a partial differential operator with coefficients in $\widetilde{\mathbb{C}}$ and let $N(P)$ the set of all zeros of $P$ in $\widetilde{\mathbb{C}}^{n}$ with imaginary part of log-type. If $P(D)$ is $\mathcal{G}^{\infty}$ hypoelliptic then

$$
\mathrm{v}(|\operatorname{Re} \zeta|) \geq 0
$$

for all $\zeta \in N(P)$.

Proof. Let $\Omega$ be an open subset of $\mathbb{R}^{n}$. Under the assumption of $\mathcal{G}^{\infty}$-hypoellipticity we know that the set $\operatorname{Ker} P:=\{u \in \mathcal{G}(\Omega): P(D) u=0\}$ is contained in $\mathcal{G}^{\infty}(\Omega)$ and therefore in $\mathcal{G}(\Omega)$. Since $\mathcal{G}(\Omega)$ is a Fréchet $\widetilde{\mathbb{C}}$-module and $P(D)$ is a continuous map from $\mathcal{G}(\Omega)$ into itself we conclude that $\operatorname{Ker} P$ is a Fréchet $\widetilde{\mathbb{C}}$-module when endowed with the topology of $\mathcal{G}(\Omega)$.

We can now define the map

$$
F: \operatorname{Ker} P \rightarrow \mathcal{G}^{\infty}(\Omega): u \rightarrow \Delta u .
$$


An application of the closed graph theorem allows to prove that $F$ is continuous. We have to verify that the graph of $F$ is sequentially closed. Let $(u, v) \in \overline{\operatorname{Graph}(F)}, u_{n} \rightarrow u$ in $\operatorname{Ker} P$ and $\Delta u_{n} \rightarrow v$ in $\mathcal{G}^{\infty}(\Omega)$. By continuity properties we have that $\Delta u_{n} \rightarrow \Delta u$ in $\mathcal{G}(\Omega)$. Since at the same time $\Delta u_{n} \rightarrow v$ in $\mathcal{G}(\Omega)$ we conclude that $v=\Delta u$. Hence, $(u, v) \in \operatorname{Graph}(F)$.

In terms of valuations the continuity of $F$ can be expressed as follows: for all $K \Subset \Omega$ there exists $L \Subset \Omega, j \in \mathbb{N}$ and $c \in \mathbb{R}$ such that

$$
\mathrm{v}_{\mathcal{G}}^{\infty}{ }_{(K)}(F u) \geq c+\mathrm{v}_{L, j}(u)
$$

for all $u \in \operatorname{Ker} P$. This means that for all $u \in \operatorname{Ker} P$ and for all $m \in \mathbb{N}$,

$$
\mathrm{v}_{\infty, K}\left(\Delta^{m}(F u)\right) \geq c+\mathrm{v}_{L, j}(u),
$$

where $\mathrm{v}_{\infty, K}\left(\Delta^{m}(F u)\right)=\mathrm{v}\left(\left[\left(\sup _{x \in K} \Delta^{m} F u_{\varepsilon}(x)\right)_{\varepsilon}\right]\right)$.

Let $\zeta \in N(P)$. As observed above $w=\mathrm{e}^{i x \zeta} \in \mathcal{G}(\Omega)$ and since $P(D)\left(\mathrm{e}^{i x \zeta}\right)=P(\zeta)\left(\mathrm{e}^{i x \zeta}\right)$ we have that $w \in \operatorname{Ker} P$. Moreover, $F w(x)=-\zeta^{2} \mathrm{e}^{i x \zeta}$ for $\zeta^{2}=\zeta_{1}^{2}+\ldots+\zeta_{n}^{2}$. The choice of $w$ in (14) yields

$$
\mathrm{v}_{\infty, K}\left(\left(\zeta^{2}\right)^{m} \mathrm{e}^{i x \zeta}\right) \geq c+\mathrm{v}_{L, j}\left(\mathrm{e}^{i x \zeta}\right) \geq c+\mathrm{v}\left((1+|\zeta|)^{j}\right)+\mathrm{v}\left(\mathrm{e}^{a_{1}|\operatorname{Im} \zeta|}\right),
$$

where $a_{1}=\sup _{x \in L}|x|$. Since for $a_{2}=\sup _{x \in K}|x|$

$$
\mathrm{v}\left(\left|\zeta^{2}\right|^{m} \mathrm{e}^{-a_{2}|\operatorname{Im} \zeta|}\right) \geq \mathrm{v}_{\infty, K}\left(\left(\zeta^{2}\right)^{m} \mathrm{e}^{i x \zeta}\right)
$$

we obtain that

$$
\mathrm{v}\left(\left|\zeta^{2}\right|^{m}\right) \geq c+\mathrm{v}\left((1+|\zeta|)^{j}\right)+2 \mathrm{v}\left(\mathrm{e}^{a|\operatorname{Im} \zeta|}\right),
$$

with $a=a_{1}+a_{2}$. The constants $c, a$ and $j$ in (15) do not depend on $\zeta \in N(P)$ and $m$. This leads to

$$
\mathrm{v}\left(\left|\zeta^{2}\right|\right) \geq \frac{c}{m}+\frac{\mathrm{v}\left((1+|\zeta|)^{j}\right)}{m}+\frac{2 \mathrm{v}\left(\mathrm{e}^{a|\operatorname{Im} \zeta|}\right)}{m}
$$

and therefore to $\mathrm{v}\left(\left|\zeta^{2}\right|\right) \geq 0$. Finally, from the equality

$$
\zeta^{2}=\zeta_{1}^{2}+\ldots+\zeta_{n}^{2}=|\operatorname{Re} \zeta|^{2}+2 i \operatorname{Re} \zeta \operatorname{Im} \zeta-|\operatorname{Im} \zeta|^{2}
$$

we have that $\mathrm{v}\left(\left.|| \operatorname{Re} \zeta\right|^{2}-|\operatorname{Im} \zeta|^{2} \mid\right) \geq \mathrm{v}\left(\left|\zeta^{2}\right|\right) \geq 0$. We can now conclude that

$$
\mathrm{v}\left(|\operatorname{Re} \zeta|^{2}\right) \geq \min \left\{\mathrm{v}\left(\left.|| \operatorname{Re} \zeta\right|^{2}-|\operatorname{Im} \zeta|^{2} \mid\right), \mathrm{v}\left(|\operatorname{Im} \zeta|^{2}\right)\right\}=0 .
$$

This necessary condition of $\mathcal{G}^{\infty}$-hypoellipticity has been also obtained, via a completely different method, in [11, Theorem 4.9]. Here, by means of analytic estimates involving the Laplace-Fourier transform of generalized functions in $\mathcal{G}_{\mathrm{c}}\left(\mathbb{R}^{n}\right)$ and functionals in $\mathcal{L}\left(\mathcal{G}\left(\mathbb{R}^{n}\right), \widetilde{\mathbb{C}}\right)$ and by making use of the characterizations of $\mathcal{G}$ - and $\mathcal{G}^{\infty}$-hypoellipticity provided by Theorem 12 , the author deduces (13) again in case of $\mathcal{G}^{\infty}$-hypoellipticity and succeeds to formulate a necessary condition for $\mathcal{G}$-hypoellipticity. Here we improve Theorem 4.9 of [11] by stating the assertions via the order relation $\leq$ in $\widetilde{\mathbb{R}}$.

THEOREM 15. Let $P(D)$ be a partial differential operator of order $m$ with coefficients in $\widetilde{\mathbb{C}}$ such that $\widetilde{P}$ is invertible in some point of $\mathbb{R}^{n}$ and let $N(P)$ the set of all zeros of $P$ in $\widetilde{\mathbb{C}}^{n}$ with imaginary part of log-type. 
(i) If $P(D)$ is $\mathcal{G}$-hypoelliptic then there exist $c \in \widetilde{\mathbb{R}}$ strictly nonzero and a real $a>0$ such that

$$
|\zeta| \leq c \mathrm{e}^{a|\operatorname{Im} \zeta|}
$$

for all $\zeta \in N(P)$.

(ii) If $P(D)$ is $\mathcal{G}^{\infty}$-hypoelliptic then there exist $c \in \widetilde{\mathbb{R}}$ strictly nonzero and a real $a>0$ such that

$$
|\zeta|^{M} \leq c \mathrm{e}^{a|\operatorname{Im} \zeta|}
$$

for all $M \in \mathbb{N}$ and for all $\zeta \in N(P)$.

Proof. (i) Under the hypothesis of invertibility on $\widetilde{P}$ we know that if $P(D)$ is $\mathcal{G}$-hypoelliptic then it admits a $\mathcal{G}$-parametrix in $\mathcal{L}_{\mathrm{b}}\left(\mathcal{G}_{\mathrm{c}}\left(\mathbb{R}^{n}\right), \widetilde{\mathbb{C}}\right)$ which belongs to $\mathcal{G}$ outside the origin. Making use of a cut-off function $\psi$ identically 1 in a neighborhood of the origin we can assume that there exists $F \in \mathcal{L}_{\mathrm{b}}\left(\mathcal{G}\left(\mathbb{R}^{n}\right), \widetilde{\mathbb{C}}\right)$ and $v \in \mathcal{G}_{\mathrm{c}}\left(\mathbb{R}^{n}\right)$ such that

$$
P(D) F=\iota_{d}(\delta)+v
$$

in $\mathcal{L}\left(\mathcal{G}\left(\mathbb{R}^{n}\right), \widetilde{\mathbb{C}}\right)$. Let now $\zeta \in N(P)$. As observed above $\mathrm{e}^{-i x \zeta} \in \mathcal{G}\left(\mathbb{R}_{x}^{n}\right)$ and therefore

$$
P(D) F\left(\mathrm{e}^{-i \cdot \zeta}\right)=1+v\left(\mathrm{e}^{-i \cdot \zeta}\right) .
$$

At the level of representatives this means that

$$
P_{\varepsilon}\left(\zeta_{\varepsilon}\right) \widehat{F_{\varepsilon}}\left(\zeta_{\varepsilon}\right)=1+\widehat{v_{\varepsilon}}\left(\zeta_{\varepsilon}\right)+n_{\varepsilon}
$$

where $\left(\zeta_{\varepsilon}\right)$ is a representative of $\zeta$ such that $\left(\mathrm{e}^{\left|\operatorname{Im} \zeta_{\varepsilon}\right|}\right)_{\varepsilon} \in \mathcal{E}_{M},\left(n_{\varepsilon}\right)_{\varepsilon} \in \mathcal{N}$ and ^ denotes the Laplace-Fourier transform. The net of distributions $\left(F_{\varepsilon}\right)_{\varepsilon} \in \mathcal{E}^{\prime}\left(\mathbb{R}^{n}\right)^{(0,1]}$ fulfills the following condition:

$$
\exists K \Subset \mathbb{R}^{n} \exists j \in \mathbb{N} \exists N \in \mathbb{N} \exists \eta \in(0,1] \forall u \in \mathcal{C}^{\infty}\left(\mathbb{R}^{n}\right) \quad\left|F_{\varepsilon}(u)\right| \leq \varepsilon^{-N} \sup _{|\alpha| \leq j, x \in K}\left|\partial^{\alpha} u(x)\right| .
$$

Hence for all $\varepsilon \in(0, \eta]$ we obtain

$$
\left|\widehat{F_{\varepsilon}}\left(\zeta_{\varepsilon}\right)\right| \leq \varepsilon^{-N}\left(1+\left|\zeta_{\varepsilon}\right|\right)^{j} \mathrm{e}^{b\left|\operatorname{Im}\left(\zeta_{\varepsilon}\right)\right|},
$$

where $b$ depends only on the compact set $K$. It follows that the net $\left(\widehat{F_{\varepsilon}}\left(\zeta_{\varepsilon}\right)\right)_{\varepsilon}$ is moderate and since $P(\zeta)=0$ in $\widetilde{\mathbb{C}}$ we conclude that

$$
\widehat{v_{\varepsilon}}\left(\zeta_{\varepsilon}\right)=-1+n_{\varepsilon}^{\prime},
$$

where $\left(n_{\varepsilon}^{\prime}\right)_{\varepsilon} \in \mathcal{N}$. Assuming that the generalized function $v$ has $\operatorname{supp} v \subseteq\{x:|x|<a\}$ from Proposition 2 we have that

$$
\forall M \in \mathbb{N} \exists N \in \mathbb{N} \exists \eta \in(0,1] \forall \varepsilon \in(0, \eta] \forall \zeta \in \mathbb{C}^{n} \quad\left|\widehat{v}_{\varepsilon}(\zeta)\right| \leq \varepsilon^{-N}(1+|\zeta|)^{-M} \mathrm{e}^{a|\operatorname{Im} \zeta|} .
$$

This combined with (18) leads to

$$
\left|-1+n_{\varepsilon}^{\prime}\right| \leq \varepsilon^{-N}\left(1+\left|\zeta_{\varepsilon}\right|\right)^{-1} \mathrm{e}^{a\left|\operatorname{Im} \zeta_{\varepsilon}\right|},
$$

where $N$ does not depend on $\zeta=\left[\left(\zeta_{\varepsilon}\right)_{\varepsilon}\right] \in N(P)$. Choosing $\eta$ small enough such that $\left|-1+n_{\varepsilon}^{\prime}\right| \geq 1 / 2$ for all $\varepsilon \in(0, \eta]$ we can write

$$
\left|\zeta_{\varepsilon}\right| \leq 2 \varepsilon^{-N} \mathrm{e}^{a\left|\operatorname{Im} \zeta_{\varepsilon}\right|}
$$


(19) proves that there exist a strictly nonzero $c \in \widetilde{\mathbb{R}}$ and some real $a>0$ such that

$$
|\zeta| \leq c \mathrm{e}^{a|\operatorname{Im} \zeta|}
$$

for all $\zeta \in N(P)$.

(ii) If the operator $P(D)$ is $\mathcal{G}^{\infty}$-hypoellptic then we find $F \in \mathcal{L}_{\mathrm{b}}\left(\mathcal{G}\left(\mathbb{R}^{n}\right), \widetilde{\mathbb{C}}\right)$ and $v \in$ $\mathcal{G}_{\mathrm{c}}^{\infty}\left(\mathbb{R}^{n}\right)$ such that $P(D) F=\iota_{d}(\delta)+v$ in $\mathcal{L}\left(\mathcal{G}\left(\mathbb{R}^{n}\right), \widetilde{\mathbb{C}}\right)$. Moreover, since $v$ is $\mathcal{G}^{\infty}$-regular from Proposition 2 we obtain that

$$
\exists N \in \mathbb{N} \forall M \in \mathbb{N} \exists \eta \in(0,1] \forall \varepsilon \in(0, \eta] \forall \zeta \in \mathbb{C}^{n} \quad\left|\widehat{v}_{\varepsilon}(\zeta)\right| \leq \varepsilon^{-N}(1+|\zeta|)^{-M} \mathrm{e}^{a|\operatorname{Im} \zeta|},
$$

with $\operatorname{supp} v \subseteq\{x:|x|<a\}$. Arguments analogous to the ones adopted in the first case yields that the assertion

$$
\exists N \in \mathbb{N} \forall M \in \mathbb{N} \exists \eta \in(0,1] \forall \varepsilon \in(0, \eta] \quad\left(1+\left|\zeta_{\varepsilon}\right|\right)^{M} \leq 2 \varepsilon^{-N} \mathrm{e}^{a\left|\operatorname{Im} \zeta_{\varepsilon}\right|},
$$

holds for all $\zeta \in N(P)$ with $N$ and $a$ independent of $\zeta$. Therefore, there exists $c \in \widetilde{\mathbb{R}}$ strictly nonzero and $a>0$ such that

$$
|\zeta|^{M} \leq c \mathrm{e}^{a|\operatorname{Im} \zeta|}
$$

for all $M \in \mathbb{N}$ and for all $\zeta \in N(P)$.

It is clear that the assertions of Theorem 4.9 in [11] follow from (16) and (17) respectively. Choosing $M=1$ we see that estimate (17) is consistent with the well-known Hörmander necessary condition for hypoellipticity. More precisely, when $P(D)$ has classical coefficients one can take $F \in \mathcal{E}^{\prime}\left(\mathbb{R}^{n}\right), v \in \mathcal{C}_{\mathrm{c}}^{\infty}\left(\mathbb{R}^{n}\right)$ and therefore $c \in \mathbb{R}$.

5. Sufficient conditions for $\mathcal{G}$ - and $\mathcal{G}^{\infty}$-hypoellipticity. In this section we investigate the generalized polynomial $P(\xi)=\sum_{|\alpha| \leq m} c_{\alpha} \xi^{\alpha}$ more deeply and provide sufficient conditions for $\mathcal{G}$ - and $\mathcal{G}^{\infty}$-hypoellipticity. The classical characterization of hypoelliptic polynomials obtained by Hörmander in [16, Theorem 11.1.3] inspires Theorem 17 and Proposition 18 . We begin by recalling a technical lemma which will be employed in the sequel and whose proof can be found in [11].

Lemma 16. Let $\varphi \in \mathcal{C}^{\infty}\left(\mathbb{R}^{n}\right)$ such that $\varphi(\xi)=0$ for $|\xi| \leq 1$ and $\varphi(\xi)=1$ for $|\xi| \geq 2$ and let $\left(s_{\varepsilon}\right)_{\varepsilon}$ be a net of positive real numbers different from zero.

(i) If $\left(s_{\varepsilon}\right)_{\varepsilon},\left(s_{\varepsilon}^{-1}\right)_{\varepsilon} \in \mathcal{E}_{M}$ then $\left(\varphi\left(\xi / s_{\varepsilon}\right)-1\right)_{\varepsilon} \in \mathcal{E}_{\mathscr{S}}\left(\mathbb{R}^{n}\right)$;

(ii) If $\left(s_{\varepsilon}\right)_{\varepsilon}$ is a slow scale net with $\inf _{\varepsilon} s_{\varepsilon}>0$ then $\left(\varphi\left(\xi / s_{\varepsilon}\right)-1\right)_{\varepsilon} \in \mathcal{E}_{\mathscr{S}}^{\infty}\left(\mathbb{R}^{n}\right)$.

Before stating Theorem 17 we recall that $r \in \widetilde{\mathbb{R}}$ is slow scale-invertible if there exists a slow scale net $\left(\omega_{\varepsilon}\right)_{\varepsilon}$ and a representative $\left(r_{\varepsilon}\right)_{\varepsilon}$ of $r$ such that $\left|r_{\varepsilon}\right| \geq \omega_{\varepsilon}^{-1}$ for $\varepsilon$ small enough.

\section{THEOREM 17.}

(i) Let $P(D)=\sum_{|\alpha| \leq m} c_{\alpha} D^{\alpha}$ such that one of the coefficients of the principal part is invertible. If there exists a representative $\left(P_{\varepsilon}\right)_{\varepsilon}$ of $P, \delta>0$ and some positive moderate nets $\left(c_{\varepsilon}\right)_{\varepsilon}$ and $\left(R_{\varepsilon}\right)_{\varepsilon}$ and some $\eta \in(0,1]$ such that

$$
\left|P_{\varepsilon}^{(\alpha)}(\xi)\right| \leq c_{\varepsilon}|\xi|^{-\delta|\alpha|}\left|P_{\varepsilon}(\xi)\right|
$$

for all $|\alpha| \leq m,|\xi| \geq R_{\varepsilon}$ and $\varepsilon \in(0, \eta]$, then the operator $P(D)$ is $\mathcal{G}$-hypoelliptic. 
(ii) If one of the coefficients of the principal part of $P(D)$ is slow scale invertible and there exists a representative $\left(P_{\varepsilon}\right)_{\varepsilon}$ as in $(i)$ with $\delta>0$ and $\left(c_{\varepsilon}\right)_{\varepsilon}$ and $\left(R_{\varepsilon}\right)_{\varepsilon}$ slow scale nets, then the operator $P(D)$ is $\mathcal{G}^{\infty}$-hypoelliptic.

Proof. (i) We begin by proving that (20) implies an estimate from below on $\left|P_{\varepsilon}(\xi)\right|$. Let $c_{\bar{\alpha}}$ be an invertible coefficient of the principal part of $P$. Since,

$$
P_{\varepsilon}^{\bar{\alpha}}(\xi)=c_{\bar{\alpha}} \bar{\alpha} !
$$

we obtain from (20) the estimate

$$
c_{\varepsilon}|\xi|^{-\delta|\bar{\alpha}|}\left|P_{\varepsilon}(\xi)\right| \geq c_{\bar{\alpha}} \bar{\alpha} !,
$$

valid for $|\xi| \geq R_{\varepsilon}$ and $\varepsilon \in(0, \eta]$. On the other hand, we can take $\left(c_{\varepsilon}\right)$ strictly nonzero and conclude that

$$
\left|P_{\varepsilon}(\xi)\right| \geq c_{0, \varepsilon}|\xi|^{\delta m}
$$

for some strictly nonzero net $\left(c_{0, \varepsilon}\right)_{\varepsilon},|\xi| \geq R_{\varepsilon}$ and $\varepsilon \in(0, \eta]$. Since $P^{(\bar{\alpha})}(\xi)$ is invertible for every $\xi$ we have that $\widetilde{P}(\xi)$ is invertible as well. We can therefore make use of Theorem 12 in order to prove the $\mathcal{G}$-hypoellipticity of $P(D)$. More precisely, we will show that the combination of (20) and (21) allows to construct a $\mathcal{G}$-parametrix $F \in \mathcal{L}_{\mathrm{b}}\left(\mathcal{G}_{\mathrm{c}}\left(\mathbb{R}^{n}\right), \widetilde{\mathbb{C}}\right)$ of $P(D)$ which belongs to $\mathcal{G}$ outside the origin. Let us take $\left(R_{\varepsilon}\right)_{\varepsilon}$ strictly nonzero and let $\varphi$ be a function in $\mathcal{C}^{\infty}\left(\mathbb{R}^{n}\right)$ as in the previous lemma. We define the net $\left(S_{\varepsilon}\right)_{\varepsilon}$ as

$$
\frac{\varphi\left(R_{\varepsilon}{ }^{-1} \xi\right)}{P_{\varepsilon}(\xi)}
$$

for $\varepsilon \in(0, \eta]$ and 0 otherwise. From (21) it follows that $\left(S_{\varepsilon}\right)_{\varepsilon}$ determines a basic functional in $\mathcal{L}\left(\mathcal{G}_{\mathscr{S}}\left(\mathbb{R}^{n}\right), \widetilde{\mathbb{C}}\right)$. Hence, $\mathcal{F}^{-1} S \in \mathcal{L}_{\mathrm{b}}\left(\mathcal{G}_{\mathscr{S}}\left(\mathbb{R}^{n}\right), \widetilde{\mathbb{C}}\right)$ and $F(u):=\mathcal{F}^{-1} S(u), u \in \mathcal{G}_{\mathrm{c}}\left(\mathbb{R}^{n}\right)$, is a basic functional in $\mathcal{L}\left(\mathcal{G}_{\mathrm{c}}\left(\mathbb{R}^{n}\right), \widetilde{\mathbb{C}}\right)$. This is a $\mathcal{G}$-parametrix of $P(D)$. Indeed, the functional

$$
P(D) F(u)-\iota_{d}(\delta) u=S\left(\mathcal{F}^{-1}\left({ }^{t} P(D) u\right)\right)-\iota_{d}\left(\mathcal{F}^{-1} 1\right)(u)
$$

on $\mathcal{G}_{\mathrm{c}}\left(\mathbb{R}^{n}\right)$ can be represented by the integral

$$
\int_{\mathbb{R}^{n}} \mathcal{F}_{\xi \rightarrow x}^{-1}\left(\varphi\left(R_{\varepsilon}^{-1}(\xi)-1\right)(x) u_{\varepsilon}(x) d x .\right.
$$

Since Lemma 16(i) implies that $v:=\left(\mathcal{F}_{\xi \rightarrow x}^{-1}\left(\varphi\left(R_{\varepsilon}^{-1} \xi\right)-1\right)\right)_{\varepsilon}+\mathcal{N}\left(\mathbb{R}^{n}\right)$ is a well-defined element of $\mathcal{G}\left(\mathbb{R}^{n}\right)$, we conclude that $P(D) F-\iota_{d}(\delta) \in \mathcal{G}\left(\mathbb{R}^{n}\right)$. It remains to prove that $\operatorname{sing} \operatorname{supp}_{\mathcal{G}} F \subseteq\{0\}$. In detail, this means to investigate the regularity properties of the net of distributions

$$
\mathcal{F}^{-1} S_{\varepsilon}=\mathcal{F}^{-1}\left(\frac{\varphi\left(R_{\varepsilon}{ }^{-1} \xi\right)}{P_{\varepsilon}(\xi)}\right) .
$$

We begin by observing that $\partial_{\xi}^{\alpha} \frac{1}{P_{\varepsilon}(\xi)}$ is a finite sum of terms of the type

$$
c_{\beta_{1}, \ldots, \beta_{j}} \frac{P_{\varepsilon}^{\left(\beta_{1}\right)}(\xi) P_{\varepsilon}^{\left(\beta_{2}\right)}(\xi) \ldots P_{\varepsilon}^{\left(\beta_{j}\right)}(\xi)}{P_{\varepsilon}^{j+1}(\xi)},
$$

where $\left|\beta_{1}+\ldots+\beta_{j}\right|=|\alpha|$. From the estimates (20) and (21) it follows that the net of distributions $\left(F_{\varepsilon}\right)_{\varepsilon}:=\left(\mathcal{F}^{-1} S_{\varepsilon}\right)_{\varepsilon}$ in $\mathscr{S}^{\prime}\left(\mathbb{R}^{n}\right)^{(0,1]}$ which determines $F$ satisfies the following 
properties: for every $\alpha \in \mathbb{N}^{n}$ and every $\varepsilon \in(0,1]$

$$
x^{\alpha} F_{\varepsilon} \in \mathcal{C}^{q}\left(\mathbb{R}^{n}\right),
$$

with $q \leq \delta(m+|\alpha|)-n-1$. In particular, for all $K \Subset \mathbb{R}^{n}$ and every $\beta \in \mathbb{N}^{n}$ with $|\beta| \leq q$ there exists $N \in \mathbb{N}$ such that

$$
\sup _{x \in K}\left|\partial^{\beta}\left(x^{\alpha} F_{\varepsilon}\right)(x)\right|=O\left(\varepsilon^{-N}\right) .
$$

This means that away from 0 the net $\left(F_{\varepsilon}\right)_{\varepsilon}$ is moderate, i.e, $\left(\left.F_{\varepsilon}\right|_{\mathbb{R}^{n} \backslash 0}\right)_{\varepsilon} \in \mathcal{E}_{M}\left(\mathbb{R}^{n} \backslash 0\right)$. As a consequence sing $\operatorname{supp}_{\mathcal{G}} F \subseteq\{0\}$.

(ii) It is not restrictive to assume that $\inf _{\varepsilon} c_{\varepsilon}$ and $\inf _{\varepsilon} R_{\varepsilon}$ are positive. We obtain under the assumptions of $(i i)$ that $(21)$ holds for some net $\left(c_{0, \varepsilon}\right)_{\varepsilon}$ which is the inverse of a slow scale net. The previous lemma entails that $v:=\left(\mathcal{F}_{\xi \rightarrow x}^{-1}\left(\varphi\left(R_{\varepsilon}^{-1} \xi\right)-1\right)\right)_{\varepsilon}+\mathcal{N}\left(\mathbb{R}^{n}\right)$ is a well-defined element of $\mathcal{G}^{\infty}\left(\mathbb{R}^{n}\right)$. Therefore, $F$ is a $\mathcal{G}^{\infty}$-parametrix of $P(D)$. Finally, arguing as above we see that for all $\alpha, \beta$ there exists a slow scale net $\left(\omega_{\varepsilon}\right)_{\varepsilon}$ such that

$$
\sup _{x \in K}\left|\partial^{\beta}\left(x^{\alpha} F_{\varepsilon}\right)(x)\right|=O\left(\omega_{\varepsilon}\right) .
$$

We conclude that the net $\left(F_{\varepsilon}\right)_{\varepsilon}$ is $\mathcal{G}^{\infty}$-moderate on $\mathbb{R}^{n} \backslash\{0\}$, i.e, sing $\operatorname{supp}_{\mathcal{G}^{\infty}} F \subseteq\{0\}$.

In the following proposition we see how condition (20) is connected to a geometric property of the hypersurface $\left\{\zeta \in \mathbb{C}^{n}: P_{\varepsilon}(\zeta)=0\right\}$. Let $\xi \in \mathbb{R}^{n}$ and

$$
d_{\varepsilon}(\xi):=\inf _{\zeta \in \mathbb{C}^{n}}\left|\xi-P_{\varepsilon}(\zeta)\right| .
$$

Since Lemma 11.1.4 in [16] is valid for all polynomials on $\mathbb{R}^{n}$ with degree $\leq m$, we know that there exists a constant $C>0$ such that

$$
C^{-1} \leq d_{\varepsilon}(\xi) \sum_{|\alpha| \leq m}\left|\frac{P_{\varepsilon}^{(\alpha)}(\xi)}{P_{\varepsilon}(\xi)}\right|^{\frac{1}{|\alpha|}} \leq C
$$

for all $\varepsilon \in(0,1]$ and for all $\xi \in \mathbb{R}^{n}$ such that $P_{\varepsilon}(\xi) \neq 0$. The inequality (22) is essential for Proposition 18.

Proposition 18.

(i) Let $P(D)=\sum_{|\alpha|<m} c_{\alpha} D^{\alpha}$ such that one of the coefficients of the principal part is invertible. Then, the condition on $P$ of Theorem $17(i)$ is equivalent to the following: there exist a representative $\left(P_{\varepsilon}\right)_{\varepsilon}$ of $P, \delta>0$ and some moderate nets $\left(c_{\varepsilon}\right)_{\varepsilon}$ and $\left(R_{\varepsilon}\right)_{\varepsilon}$ such that, for $d_{\varepsilon}(\xi)$ defined as above,

$$
|\xi|^{\delta} \leq c_{\varepsilon} d_{\varepsilon}(\xi)
$$

for all $\xi$ with $|\xi| \geq R_{\varepsilon}$ and for all $\varepsilon$ in a small enough interval $(0, \eta]$.

(ii) Let $P(D)=\sum_{|\alpha|<m} c_{\alpha} D^{\alpha}$ such that one of the coefficients of the principal part is slow scale invertible. Then, the condition on $P$ of Theorem 17(ii) is equivalent to the following: there exist a representative $\left(P_{\varepsilon}\right)_{\varepsilon}$ of $P, \delta>0$ and some slow scale nets $\left(c_{\varepsilon}\right)_{\varepsilon}$ and $\left(R_{\varepsilon}\right)_{\varepsilon}$ such that, for $d_{\varepsilon}(\xi)$ defined as above,

$$
|\xi|^{\delta} \leq c_{\varepsilon} d_{\varepsilon}(\xi)
$$


for all $\xi$ with $|\xi| \geq R_{\varepsilon}$ and for all $\varepsilon$ in a small enough interval $(0, \eta]$.

Proof. ( $i$ ) We assume that (23) holds. The inequality (22) yields for $|\xi| \geq R_{\varepsilon}, \varepsilon \in(0, \eta]$ and $|\alpha| \leq m$,

$$
\left|\frac{P_{\varepsilon}^{(\alpha)}(\xi)}{P_{\varepsilon}(\xi)}\right| \leq C^{|\alpha|} c_{\varepsilon}^{|\alpha|}|\xi|^{-\delta|\alpha|},
$$

where $\left(C^{|\alpha|} c_{\varepsilon}^{|\alpha|}\right)_{\varepsilon} \in \mathcal{E}_{M}$. Conversely, the condition on $\left(P_{\varepsilon}\right)_{\varepsilon}$ of Theorem 17(i) combined with (22) entails for $|\xi| \geq R_{\varepsilon}$ and $\varepsilon \in(0, \eta]$ the estimate

$$
d_{\varepsilon}(\xi) \sum_{|\alpha| \leq m}\left|\frac{P_{\varepsilon}^{(\alpha)}(\xi)}{P_{\varepsilon}(\xi)}\right|^{\frac{1}{|\alpha|}} \leq C \sum_{|\alpha| \leq m} c_{\varepsilon}^{\frac{1}{|\alpha|}}|\xi|^{-\delta} \leq c_{\varepsilon}^{\prime}|\xi|^{-\delta},
$$

where $\left(c_{\varepsilon}^{\prime}\right)_{\varepsilon} \in \mathcal{E}_{M}$.

(ii) We argue as above with $\left(c_{\varepsilon}\right)_{\varepsilon},\left(c_{\varepsilon}^{\prime}\right)_{\varepsilon}$ and $\left(R_{\varepsilon}\right)_{\varepsilon}$ slow scale nets.

6. Generalized elliptic operators. We finally deal with the special class of $\mathcal{G}$ - and $\mathcal{G}^{\infty}$-elliptic operators.

DeFinition 19.

A partial differential operator $P(D)$ of order $m$ with coefficients in $\widetilde{\mathbb{C}}$ is said to be $\mathcal{G}$-elliptic if the generalized number

$$
\left[\left(\inf _{\xi \in \mathbb{R}^{n},|\xi|=1}\left|P_{m, \varepsilon}(\xi)\right|\right)_{\varepsilon}\right]
$$

is invertible.

It is said to be $\mathcal{G}^{\infty}$-elliptic if the generalized number in (25) is slow scale-invertible. Since, given two different representatives $\left(P_{\varepsilon}\right)_{\varepsilon}$ and $\left(P_{\varepsilon}^{\prime}\right)_{\varepsilon}$ of $P$ the inequality

$$
\left|\inf _{|\xi|=1}\right| P_{m, \varepsilon}(\xi)\left|-\inf _{|\xi|=1}\right| P_{m, \varepsilon}^{\prime}(\xi)|| \leq \sup _{|\xi|=1}\left|\left(P_{m, \varepsilon}-P_{m, \varepsilon}^{\prime}\right)(\xi)\right|
$$

holds for all $\varepsilon$, it follows that the generalized number in (25) does not depend on the choice of the representatives of the polynom $P$ but on the operator $P(D)$. In particular, Definition 19 means that for any choice of representatives of the coefficients of $P(\xi)$ the net $\left(P_{m, \varepsilon}\right)_{\varepsilon}$ satisfies the estimate

$$
\left|P_{m, \varepsilon}(\xi)\right| \geq \varepsilon^{r}, \quad|\xi|=1, \varepsilon \in(0, \eta]
$$

when $P(D)$ is $\mathcal{G}$-elliptic and the estimate

$$
\left|P_{m, \varepsilon}(\xi)\right| \geq c_{\varepsilon}^{-1}, \quad|\xi|=1, \varepsilon \in(0, \eta],
$$

with some slow scale net $\left(c_{\varepsilon}\right)_{\varepsilon}$, when $P(D)$ is $\mathcal{G}^{\infty}$-elliptic.

The results stated in the sequel are detailed proved in [11, Subsection 4.2]. LEMMA 20.

(i) Let $P(D)$ be a $\mathcal{G}$-elliptic operator of order $m$ with coefficients in $\widetilde{\mathbb{C}}$. Then there exist $M \in \mathbb{N}, a \in \mathbb{R}$ and $\eta \in(0,1]$ such that

$$
\left|P_{\varepsilon}(\xi)\right| \geq \varepsilon^{a}|\xi|^{m}
$$

for all $\xi \in \mathbb{R}^{n}$ with $|\xi| \geq \varepsilon^{-M}$ and for all $\varepsilon \in(0, \eta]$. 
(ii) If $P(D)$ is a $\mathcal{G}^{\infty}$-elliptic operator of order $m$ with coefficients in $\mathcal{G}_{\mathbb{C}}^{\text {sc }}$ then there exist two slow scale nets $\left(\omega_{\varepsilon}\right)_{\varepsilon}$ and $\left(s_{\varepsilon}\right)_{\varepsilon}$ and a constant $\eta>0$ such that

$$
\left|P_{\varepsilon}(\xi)\right| \geq \omega_{\varepsilon}^{-1}|\xi|^{m}
$$

for all $\xi \in \mathbb{R}^{n}$ with $|\xi| \geq s_{\varepsilon}$ and for all $\varepsilon \in(0, \eta]$.

Proposition 21.

(i) If $P(D)$ is a $\mathcal{G}$-elliptic operator with coefficients in $\widetilde{\mathbb{C}}$ then it satisfies the sufficient condition of $\mathcal{G}$-hypoellipticity of Theorem $17(i)$ with $\delta=1$.

(ii) If $P(D)$ is a $\mathcal{G}^{\infty}$-elliptic operator with coefficients in $\mathcal{G}_{\mathbb{C}}^{\text {sc }}$ then it satisfies the sufficient condition of $\mathcal{G}^{\infty}$-hypoellipticity of Theorem $17(i i)$ with $\delta=1$.

Proof. (i) From the definition of $\mathcal{G}$-elliptic operator it is clear that the principal symbol $P_{m}$ has an invertible coefficient. More precisely, combining the first assertion of Lemma 20 with the usual symbol estimates, we obtain

$$
\left|P_{\varepsilon}^{(\alpha)}(\xi)\right| \leq c_{\varepsilon}|\xi|^{m-|\alpha|} \leq c_{\varepsilon} \varepsilon^{-a}|\xi|^{-|\alpha|}\left|P_{\varepsilon}(\xi)\right|
$$

valid for $|\xi| \geq R_{\varepsilon}$ and $\varepsilon \in(0, \eta]$, with $\left(R_{\varepsilon}\right)_{\varepsilon} \in \mathcal{E}_{M}$ and $\eta \in(0,1]$.

(ii) Arguing as above, we have that $P_{m}$ has a slow scale invertible coefficient. In addition, there exists a slow scale net $\left(R_{\varepsilon}\right)_{\varepsilon}$ such that for $|\xi| \geq R_{\varepsilon}$ and $\varepsilon \in(0, \eta]$ the estimate

$$
\left|P_{\varepsilon}^{(\alpha)}(\xi)\right| \leq c_{\varepsilon}|\xi|^{m-|\alpha|} \leq c_{\varepsilon} \omega_{\varepsilon}|\xi|^{-|\alpha|}\left|P_{\varepsilon}(\xi)\right|,
$$

holds, with $\left(c_{\varepsilon} \omega_{\varepsilon}\right)_{\varepsilon}$ slow scale net.

A straightforward application of Theorem 17 entails the following result.

\section{THEOREM 22.}

(i) If $P(D)$ is a $\mathcal{G}$-elliptic operator with coefficients in $\widetilde{\mathbb{C}}$ then it is $\mathcal{G}$-hypoelliptic in $\mathbb{R}^{n}$.

(ii) If $P(D)$ is a $\mathcal{G}^{\infty}$-elliptic operator with coefficients in $\mathcal{G}_{\mathbb{C}}^{\text {sc }}$ then it is $\mathcal{G}^{\infty}$-hypoelliptic in $\mathbb{R}^{n}$.

\section{References}

[1] H. Biagioni, A Nonlinear Theory of Generalized Functions, Number 1421 in Lecture Notes in Math. Springer-Verlag, Berlin, 1990.

[2] J. F. Colombeau, Elementary Introduction to New Generalized Functions, North-Holland Mathematics Studies 113. Elsevier Science Publishers, 1985.

[3] J. F. Colombeau and M. Oberguggenberger, On a hyperbolic system with a compatible quadratic term: Generalized solutions, delta waves, and multiplication of distributions, Comm. Part. Diff. Eqs., 15(7):905-938, 1990.

[4] A. Delcroix, Generalized integral operators and Schwartz kernel type theorem, J. Math. Anal. Appl., 306(2):481-501, 2005.

[5] A. Delcroix and D. Scarpalezos, Topology on asymptotic algebras of generalized functions and applications, Monatsh. Math, 129(1):1-14, 2000. 
[6] C. Garetto, Pseudo-differential operators in algebras of generalized functions and global hypoellipticity, Acta Appl. Math., 80(2):123-174, 2004.

[7] C. Garetto, Topological structures in Colombeau algebras: topological $\widetilde{\mathbb{C}}-$ modules and duality theory, Acta. Appl. Math., 88(1):81-123, 2005.

[8] C. Garetto, Topological structures in Colombeau algebras: investigation of the duals of $\mathcal{G}_{\mathrm{c}}(\Omega), \mathcal{G}(\Omega)$ and $\mathcal{G}_{\mathscr{S}}\left(\mathbb{R}^{n}\right)$, Monatsh. Math., 146(3):203-226, 2005.

[9] C. Garetto, Microlocal analysis in the dual of a Colombeau algebra: generalized wave front sets and noncharacteristic regularity, New York J. Math., 12, 275-318, 2006.

[10] C. Garetto, Closed graph and open mapping theorems for topological $\widetilde{\mathbb{C}}$-modules and applications, to appear in Math. Nach., 2008.

[11] C. Garetto, Fundamental solutions in the Colombeau framework: applications to solvability and regularity theory, to appear in Acta. Appl. Math., 2008.

[12] C. Garetto, T. Gramchev and M. Oberguggenberger, Pseudodifferential operators with generalized symbols and regularity theory., Electron. J. Diff. Eqns., 2005(116):1-43, 2005.

[13] C. Garetto and G. Hörmann, Microlocal analysis of generalized functions: pseudodifferential techniques and propagation of singularities., Proc. Edinb. Math. Soc., 48(3):603-629, 2005.

[14] C. Garetto, G. Hörmann and M. Oberguggenberger, Generalized oscillatory integrals and Fourier integral operators, to appear in Proc. Edinburgh. Math. Soc., 2008.

[15] M. Grosser, M. Kunzinger, M. Oberguggenberger and R. Steinbauer, Geometric Theory of Generalized Functions, volume 537 of Mathematics and its Applications, Kluwer, Dordrecht, 2001.

[16] L. Hörmander, The Analysis of Linear Partial Differential Operators, volume II, Springer-Verlag, Berlin, 1983.

[17] G. Hörmann and M. Oberguggenberger, Elliptic regularity and solvability for partial differential equations with Colombeau coefficients, Electron. J. Diff. Eqns., 2004(14):130, 2004.

[18] G. Hörmann, M. Oberguggenberger and S. Pilipovic. Microlocal hypoellipticity of linear partial differential operators with generalized functions as coefficients., Trans. Amer. Math. Soc., 358:3363-3383, 2006.

[19] M. Nedeljkov, S. Pilipović and D. Scarpalézos, The Linear Theory of Colombeau Generalized Functions, Pitman Research Notes in Mathematics 385. Longman, Harlow, 1998.

[20] M. Oberguggenberger, Multiplication of Distributions and Applications to Partial Differential Equations, Pitman Research Notes in Mathematics 259. Longman, Harlow, 1992.

[21] L. Rodino, Linear partial differential operators in Gevrey spaces, World Scient. Publish., River Edge, NJ, 1993. 